ARTICLE

\title{
Astrocytes gate Hebbian synaptic plasticity in the striatum
}

Silvana Valtcheva ${ }^{1,2} \&$ Laurent Venance ${ }^{1,2}$

Astrocytes, via excitatory amino-acid transporter type-2 (EAAT2), are the major sink for released glutamate and contribute to set the strength and timing of synaptic inputs. The conditions required for the emergence of Hebbian plasticity from distributed neural activity remain elusive. Here, we investigate the role of EAAT2 in the expression of a major physiologically relevant form of Hebbian learning, spike timing-dependent plasticity (STDP). We find that a transient blockade of EAAT2 disrupts the temporal contingency required for Hebbian synaptic plasticity. Indeed, STDP is replaced by aberrant non-timing-dependent plasticity occurring for uncorrelated events. Conversely, EAAT2 overexpression impairs the detection of correlated activity and precludes STDP expression. Our findings demonstrate that EAAT2 sets the appropriate glutamate dynamics for the optimal temporal contingency between pre- and postsynaptic activity required for STDP emergence, and highlight the role of astrocytes as gatekeepers for Hebbian synaptic plasticity.

\footnotetext{
${ }^{1}$ Dynamics and Pathophysiology of Neuronal Networks Team, Center for Interdisciplinary Research in Biology, College de France, CNRS UMR7241/INSERM U1050, MemoLife Labex, 75005 Paris, France. ${ }^{2}$ Pierre et Marie Curie University, ED 158, 75005 Paris, France. Correspondence and requests for materials should be addressed to L.V. (email: laurent.venance@college-de-france.fr).
} 
F ast excitatory transmission at central synapses is dependent on glutamate dynamics. Astrocytes play a major role in the precise regulation of glutamate concentration in the extracellular fluid, via their high-affinity glutamate transporters (excitatory amino acid transporters, EAATs), which determine the extent of receptor stimulation by terminating the neurotransmitter signal ${ }^{1-4}$. Among the five subtypes of EAATs, the largest proportion of glutamate uptake (95\%) in the adult forebrain is mediated by the astrocytic EAAT2 (refs 5-8). Specific deletion of EAAT2 in astrocytes (which express $90 \%$ of total EAAT2) revealed that astrocytic EAAT2 contributes to most of the glutamate uptake and that specific EAAT2 deletion in neurons has to this day unidentified consequences ${ }^{8,9}$. Decreased levels of EAAT2 associated with increased ambient glutamate have been observed in neurodegenerative and psychiatric diseases $7,10,11$ and in chronic exposure to drugs of abuse ${ }^{12}$.

EAAT2 is of crucial importance in the maintenance of low glutamate concentrations and for ensuring a high signal-to-noise ratio in synaptic and extrasynaptic transmission ${ }^{4,13}$. Astrocytic glutamate uptake via EAAT2 affects both the fast component of the synaptic glutamate transient and slower components by limiting the spill-out to extrasynaptic receptors and the spillover to neighboring synapses ${ }^{13-15}$. Although, astrocytic glutamate transporters are not overwhelmed on physiological activity ${ }^{16}$, synaptic isolation is never reached ${ }^{17}$. Thus, fast removal of glutamate by astrocytes contributes to set the strength and timing of synaptic inputs by controlling periand extrasynaptic receptor activation during neuronal activity ${ }^{18}$.

According to Hebbian theory, neural networks refine their connectivity by patterned firing of action potentials in pre- and postsynaptic neurons ${ }^{19}$. Spike timing-dependent plasticity (STDP) is a synaptic Hebbian learning rule that has been the focus of considerable attention in experimental ${ }^{19,20}$ and computational $^{21,22}$ neuroscience. STDP relies on the precise order and the millisecond timing of the paired activities on either side of the synapse ${ }^{19,20}$. However, the conditions required for the emergence of STDP from distributed neural activity remain unclear.

Temporal coding via STDP may be essential for the role of the striatum in learning of motor sequences in which sensory and motor events are associated in a precise time sequence. Corticostriatal longterm plasticity provides a fundamental mechanism for the function of the basal ganglia in procedural learning ${ }^{23,24}$. MSNs act as detectors of distributed patterns of cortical and thalamic activity. Thus, the physiological or pathological regulation of EAAT2 expression should play a major role in information processing in the basal ganglia, which is based on a precise time-coding process. EAAT2 is highly expressed in the striatum ${ }^{7}$ and specific knockout of astrocytic EAAT2 leads to pathological repetitive behaviours due to corticostriatal dysfunction ${ }^{25}$. We have previously shown, by dual astrocyteneuron recordings, that EAAT2 controls corticostriatal transmission and short-term plasticity, and increases the strength of cortical input filtering by the striatum ${ }^{26}$. Here we questioned the role of astrocytes (via EAAT2) in the control of Hebbian plasticity expression, and, more specifically, corticostriatal STDP. We find that under a transient blockade of EAAT2, a non-Hebbian form of plasticity occurring for uncorrelated events replaces STDP. By contrast, EAAT2 overexpression impairs the detection of correlated pre- and postsynaptic activity by MSNs, resulting in the absence of plasticity. We demonstrate here that astrocytes, via EAAT2, set the appropriate glutamate dynamics for the emergence and the establishment of synaptic Hebbian learning rule, such as STDP.

\section{Results}

Bidirectional STDP within a narrow temporal window. We investigated the effect of EAAT2 on STDP, using whole-cell recordings from striatal medium-sized spiny neurons (MSNs) in horizontal corticostriatal brain slices from juvenile rats $^{27}$ (Fig. 1a). Baseline excitatory postsynaptic currents (EPSCs) were recorded for $10 \mathrm{~min}$ in voltage-clamp mode and then recordings were switched to current-clamp mode to pair a single excitatory postsynaptic potential (EPSP) induced by presynaptic stimulation with a single postsynaptic spike induced by a brief depolarization of the MSN (Fig. 1b). The STDP protocol involved pairing pre- and postsynaptic stimulation with a certain fixed timing interval, $\Delta t_{\text {STDP }}\left(\Delta t_{\text {STDP }}<0\right.$ indicating that postsynaptic stimulation preceded presynaptic stimulation and $\Delta t_{\mathrm{STDP}}>0$ indicating that presynaptic stimulation preceded postsynaptic stimulation), repeated 100 times at $1 \mathrm{~Hz}$. After the STDP protocol, recordings were obtained in voltage-clamp mode, and EPSCs were monitored for $1 \mathrm{~h}$.

Post- and presynaptic activities paired within a narrow time window $\left(-30<\Delta t_{\mathrm{STDP}}<+30 \mathrm{~ms}\right)$ induced bidirectional STDP in MSNs. An example of the timing-dependent longterm potentiation (t-LTP) induced by post-pre pairings $\left(\Delta t_{\mathrm{STDP}}=-12 \mathrm{~ms}\right)$ is illustrated in Fig. 1c; the mean baseline EPSC amplitude was $168 \pm 5 \mathrm{pA}$ before pairings, and increased by $324 \%$ to $711 \pm 22 \mathrm{pA} 1 \mathrm{~h}$ after pairings. Ri remained stable over this period. Conversely, pre-post pairings $\left(\Delta t_{\mathrm{STDP}}=+13 \mathrm{~ms}\right)$ induced timing-dependent long-term depression (t-LTD), as shown in the example in Fig. 1d: the mean baseline EPSC amplitude, $474 \pm 10 \mathrm{pA}$, had decreased by $33 \%$, to $318 \pm 7 \mathrm{pA}, 1 \mathrm{~h}$ after pairing. To summarize, post-pre pairings $\left(-30<\Delta t_{\text {STDP }}<0 \mathrm{~ms}\right.$ ) induced t-LTP (mean EPSC amplitude recorded $60 \mathrm{~min}$ after protocol induction: $207 \pm 35 \%$ of baseline, $P=0.0116, n=11 ; 9$ of 11 cells displayed LTP), whereas pre-post pairings $\left(0<\Delta t_{\mathrm{STDP}}<+30 \mathrm{~ms}\right)$ induced t-LTD $\quad(61 \pm 5 \%$, $P=0.0001, n=7 ; 7 / 7$ cells displayed LTD) (Fig. 1e,f,i), resulting in anti-Hebbian STDP. We have shown that GABA controls the polarity of corticostriatal STDP ${ }^{28}$ and that Hebbian ${ }^{29,30}$ or antiHebbian $^{27,31,32}$ STDP were observed, depending on whether $\mathrm{GABA}_{\mathrm{A}}$ receptor antagonists are used. The pairings for $\Delta t_{\mathrm{STDP}} \sim-30 \mathrm{~ms}$ and $\Delta t_{\mathrm{STDP}} \sim+30 \mathrm{~ms}$ did not induce plasticity ( $97 \pm 5 \%, P=0.6205, n=4$ and $105 \pm 5 \%, P=0.4670$, $n=3)$. Less correlated pairings $\left(\Delta t_{\mathrm{STDP}}<-30 \mathrm{~ms}\right.$ and $\left.\Delta t_{\mathrm{STDP}}>+30 \mathrm{~ms}\right)$ failed to induce long-term synaptic efficacy changes. Indeed, for $-250<\Delta t_{\text {STDP }}<-100 \mathrm{~ms}$ and $+100<\Delta t_{\mathrm{STDP}}<+250 \mathrm{~ms}$, we observed no plasticity $(98 \pm 6 \%$, $P=0.7931, n=7$ and $91 \pm 4 \%, P=0.1067, n=5$, respectively; Fig. 1g,i). Uncorrelated pairings up to $\pm 500 \mathrm{~ms}$, the maximum interval between the postsynaptic action potential and the presynaptic stimulation paired at $1 \mathrm{~Hz}$, also failed to induce long-term synaptic efficacy changes (103 $\pm 5 \%, P=0.4577, n=7$; Fig. 1h,i). Thus, post- and presynaptic activities paired only within a narrow temporal window, spanning $60 \mathrm{~ms}$ $\left(-30<\Delta t_{\mathrm{STDP}}<+30 \mathrm{~ms}\right)$, efficiently induce bidirectional STDP (Fig. 1i).

EAAT2 gates the polarity and temporal window of STDP. Investigation of the role of astrocytic glutamate uptake in corticostriatal STDP required the transient blocking of EAAT2 during the STDP pairings (see Methods section). We considered a pharmacological approach to be most appropriate for this purpose. We previously showed, by dual astrocyte-neuron recordings, that dihydrokainate (DHK; $300 \mu \mathrm{M})$, a selective nontransportable inhibitor of EAAT2 (ref. 33), efficiently blocked most of the transporter-mediated currents in striatal astrocytes on corticostriatal stimulation $^{26}$. Brief EAAT2 blockade with DHK for 5 min resulted in a marked depolarization of the recorded MSN in current-clamp mode in the absence of cortical stimulation $(22 \pm 2 \mathrm{mV}, P<0.0001, n=14$; Fig. $2 \mathrm{a})$. This effect was fully reversible after $15 \mathrm{~min}$ of $\mathrm{DHK}$ washout. These findings suggest 
that the slice contained sufficiently large amounts of glutamate to induce postsynaptic depolarization during EAAT2 blockade. DHK-induced depolarization involved AMPAR and type-I/II mGluR activation (Fig. 2a). Indeed, during the concomitant inhibition of AMPAR with CNQX $(20 \mu \mathrm{M})$ and of type-I/II
mGluR with MCPG $(500 \mu \mathrm{M})$ no significant depolarization was observed $(1.0 \pm 0.3 \mathrm{mV}, \quad P=0.5872, \quad n=7)$. NMDAR inhibition with D-AP5 $(50 \mu \mathrm{M})$ did not prevent DHK-induced depolarization (one-way analysis of variance (ANOVA): $P<0.0001$; post hoc Bonferroni-corrected pairwise comparisons: a

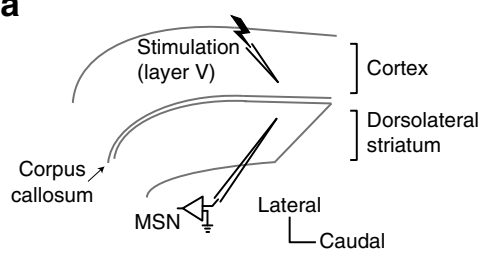

c

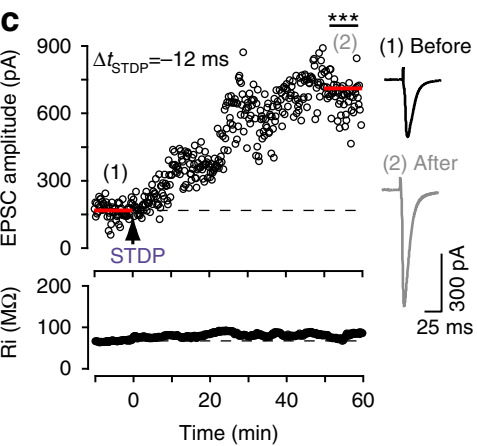

$\quad$ e $-30<\Delta t_{\mathrm{STDP}}<0 \mathrm{~ms}(n=11)$
$\quad 00<\Delta t_{\mathrm{STDP}}<+30 \mathrm{~ms}(n=7)$

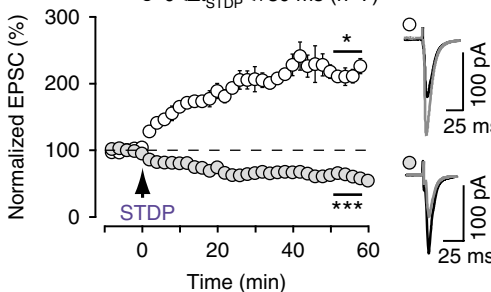

g $\quad 0-250<\Delta t_{\mathrm{STDP}}<-100 \mathrm{~ms}(n=7)$

$0+100<\Delta t_{\mathrm{STDP}}<+250 \mathrm{~ms}(n=5)$

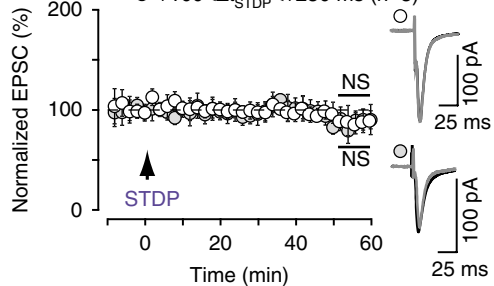

b
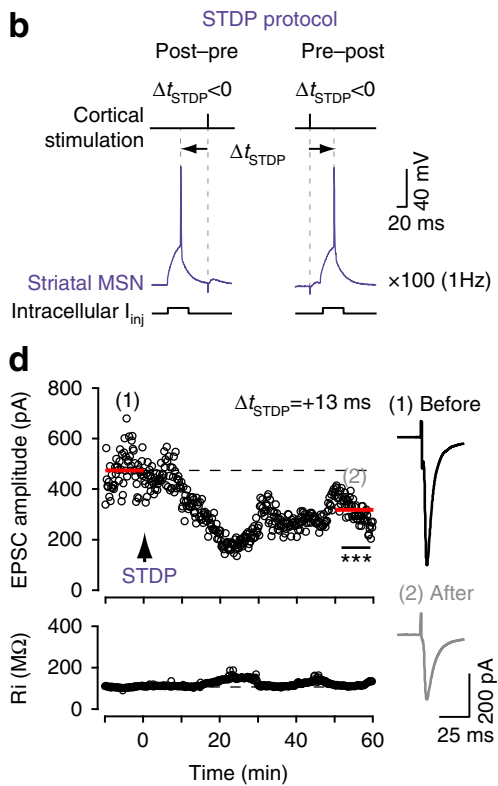

f

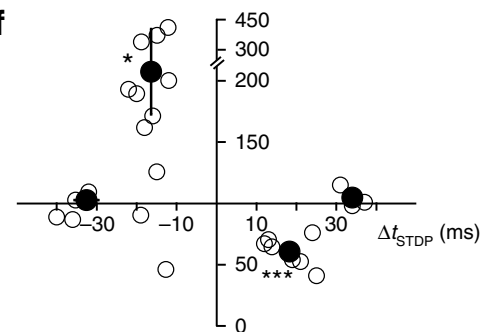

Normalized EPSC (\%)

h

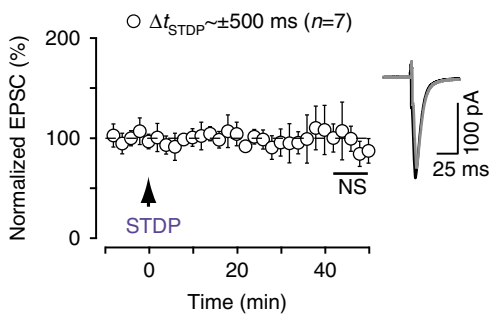

i

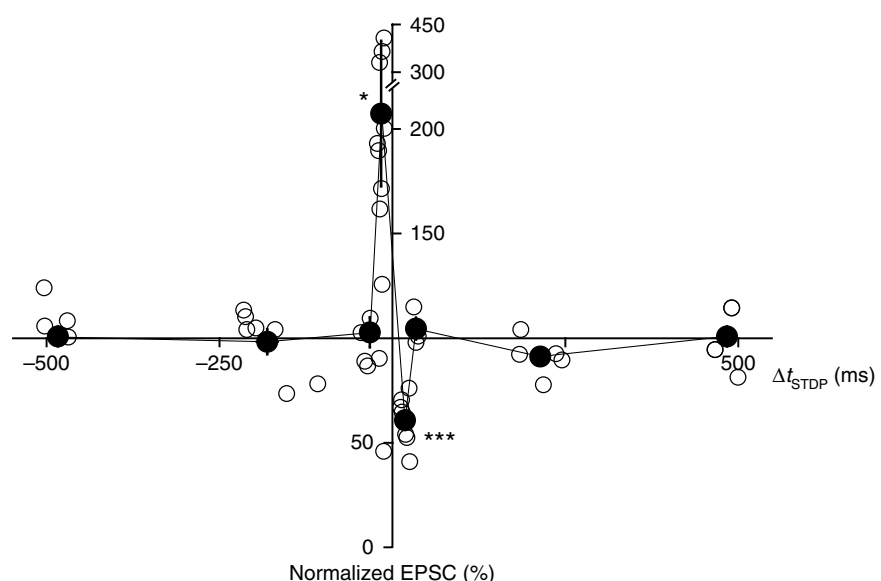


DHK-D-AP5: $\quad P>0.05, \quad$ DHK-CNQX: $\quad P<0.001, \quad$ DHK-DAP5 + CNQX + MCPG: $P<0.001$; Fig. 2a).

We then ensured that brief ( 5 min) EAAT2 blockade induced no long-term change in synaptic efficacy. A stable baseline was established over a period of $10 \mathrm{~min}$. We then applied DHK for $5 \mathrm{~min}$ without STDP pairing. As exemplified in Fig. 2b,c, we observed a transient decrease in EPSC amplitude $(65 \pm 9 \%$, $P=0.0105, n=6)$ due to AMPAR desensitization, as previously reported ${ }^{26}$, and an inward shift of $I_{\text {holding }}(-199 \pm 41 \mathrm{pA}$, $P=0.0022$; Ri was not significantly affected, $P=0.8182$; Fig. $2 c$ ). These effects were fully reversed $15 \mathrm{~min}$ after DHK removal $(93 \pm 9 \%, \quad P=0.4749$ and $11 \pm 15 \mathrm{pA}, \quad P=0.1797$, respectively; Fig. 2c). Thus, transient EAAT2 blockade with DHK was compatible with the estimation of long-term changes in synaptic efficacy.

For transient EAAT2 blockade during STDP pairings, we observed a profound change in STDP, as synaptic plasticity extended over the entire temporal window: LTD for a narrow $\Delta t_{\text {STDP }}\left(-70<\Delta t_{\text {STDP }}<+70 \mathrm{~ms}\right)$ and LTP for a broader $\Delta t_{\text {STDP }}$ $\left(-250<\Delta t_{\mathrm{STDP}}<-100 \mathrm{~ms}, \quad+100<\Delta t_{\mathrm{STDP}}<+250 \mathrm{~ms}\right.$ and $\Delta t_{\mathrm{STD}}= \pm 500 \mathrm{~ms}$ ) (Fig. 2). An example of LTD induced by post-pre pairings $\left(\Delta t_{\mathrm{STDP}}=+38 \mathrm{~ms}\right)$ under transient EAAT2 blockade with DHK $(300 \mu \mathrm{M})$ is shown in Fig. 2d; the mean baseline EPSC amplitude was $200 \pm 5 \mathrm{pA}$ before pairings and had decreased by $38 \%$, to $125 \pm 3 \mathrm{pA}, 1 \mathrm{~h}$ after pairings. Both post-pre and pre-post pairings induced LTD in a $\Delta t_{\mathrm{STDP}}$ spanning $\quad 140 \mathrm{~ms} \quad\left(-70<\Delta t_{\mathrm{STDP}}<+70 \mathrm{~ms}\right) \quad(66 \pm 6 \%$, $P=0.0005, \quad n=9 ; \quad 8 / 9$ cells displayed LTD for $70<\Delta t_{\mathrm{STDP}}<0 \mathrm{~ms}$ and $63 \pm 5 \%, P=0.0008, n=6 ; 6 / 6$ cells displayed LTD for $0<\Delta t_{\text {STDP }}<+70 \mathrm{~ms}$; Fig. 2e,f). LTD was of similar amplitude for post-pre and pre-post pairings $(P=0.7924)$. For more uncorrelated pairings $\left(\Delta t_{\mathrm{STDP}}<-70 \mathrm{~ms}\right.$ and $\Delta t_{\text {STDP }}>+70 \mathrm{~ms}$ ), LTP extended over the entire temporal window until $\pm 500 \mathrm{~ms}$. Indeed, as exemplified in Fig. $2 \mathrm{~g}$, we observed LTP for post-pairing with a $\Delta t_{\mathrm{STDP}}=-175 \mathrm{~ms}$ under transient EAAT2 blockade (mean baseline EPSC amplitude of $123 \pm 3 \mathrm{pA}$ before pairings, increasing by $66 \%$, to $203 \pm 3 \mathrm{pA}, 1 \mathrm{~h}$ after pairings). In summary, we observed LTP for $-250<\Delta t_{\mathrm{STDP}}<-70 \mathrm{~ms}$ and $+70<\Delta t_{\mathrm{STDP}}<+250 \mathrm{~ms}$ (136 $\pm 8 \%, \quad P=0.0049, \quad n=7 ; 6 / 7$ cells displayed LTP and $144 \pm 14 \%, P=0.0148, n=8 ; 6 / 8$ cells displayed LTP, respectively; Fig. 2h,j). We then assessed plasticity induction for the most uncorrelated $\Delta t_{\text {STDP }}$ that could be achieved with a pairing frequency of $1 \mathrm{~Hz}$ (that is, $\Delta t_{\mathrm{STDP}}= \pm 500 \mathrm{~ms}$ ), and we observed LTP $(136 \pm 9 \%, P=0.0085, n=7 ; 6 / 7$ cells displayed LTP; Fig. 2i,j). LTP was of similar amplitude for post-pre and pre-post pairings $(P=0.6325)$. We previously showed that bidirectional STDP was equally frequent in MSNs involved in the direct and indirect pathways ${ }^{28}$. Here, the occurrence of plasticity under EAAT2 blockade indicates a lack of segregation between the two trans-striatal pathways.

To confirm these findings, we then used another EAAT2 inhibitor, WAY-213,613, structurally distinct from DHK. Both DHK and WAY-213,613 are non-substrate competitive inhibitors (non-transported) of glutamate uptake ${ }^{33,34}$. We ensured that transient EAAT2 blockade with WAY-213,613 was reversible and, thus, compatible with the estimation of long-term changes in synaptic efficacy. The bath application of WAY-213,613 $(50 \mu \mathrm{M})$ for $5 \mathrm{~min}$ induced a transient, non-significant decrease in EPSC amplitude (with no change in Ri). This effect was fully reversible within $5 \mathrm{~min}(n=6$; Supplementary Fig. 1a-c). For transient EAAT2 blockade with WAY-213,613 (50-100 $\mu \mathrm{M})$ during STDP pairings (for $-70<\Delta t_{\mathrm{STDP}}<+70 \mathrm{~ms}$ and for $\Delta t_{\mathrm{STDP}}= \pm 200 \mathrm{~ms}$ ), we observed a profound modification of STDP (similar to that observed with DHK): LTD or no plasticity for a narrow $\Delta t_{\text {STDP }}\left(-70<\Delta t_{\text {STDP }}<+70 \mathrm{~ms}\right)$ and LTP for a broader $\Delta t_{\mathrm{STDP}}\left(\Delta t_{\mathrm{STDP}}= \pm 200 \mathrm{~ms}\right.$; Supplementary Fig. $\left.1 \mathrm{~d}-\mathrm{i}\right)$. First, for $-70<\Delta t_{\text {STDP }}<+70 \mathrm{~ms}$ with WAY-213,613 $(50 \mu \mathrm{M})$, no plasticity was observed, as exemplified in the Supplementary Fig. 1d. Both post-pre and pre-post pairings $\left(-70<\Delta t_{\mathrm{STDP}}<+70 \mathrm{~ms}\right)$ failed to induce significant plasticity (104 $\pm 5 \%, \quad P=0.4600, \quad n=5 ; \quad 1 / 5$ cells displayed LTD; Supplementary Fig. 1e). With $100 \mu \mathrm{M}$ WAY-213,613, the incidence of LTD was higher, as exemplified in the Supplementary Fig. 1f, even though, in average no significant LTD was induced for pairings at $-70<\Delta t_{\mathrm{STDP}}<+70 \mathrm{~ms}(80 \pm 11 \%, P=0.1061, n=8$; $5 / 8$ cells showed LTD; Supplementary Fig. $1 \mathrm{~g})$. LTP was observed for uncorrelated pairings $\left(\Delta t_{\mathrm{STDP}}= \pm 200 \mathrm{~ms}\right)$. An example of LTP induced by post-pre pairings $\left(\Delta t_{\mathrm{STDP}}=-200 \mathrm{~ms}\right)$ during the transient blockade of EAAT2 with WAY-213,613 $(50 \mu \mathrm{M})$ is shown in the Supplementary Fig. 1h. In summary, we observed LTP for $\Delta t_{\mathrm{STDP}}= \pm 200 \mathrm{~ms} \quad(166 \pm 21 \%, \quad P=0.0150, \quad n=8 ; \quad 7 / 8 \quad$ cells displayed LTP; Supplementary Fig. 1i).

Thus, during the transient blockade of EAAT2 with either DHK or WAY-213,613, any paired activity on either side of the synapse, regardless of $\Delta t_{\mathrm{STDP}}$, was able to modify synaptic efficacy in the long term (Fig. 2j). This finding contrasts strongly with the STDP observed in control conditions, in which EAAT2 activity was unaffected. In conclusion, the correct functioning of EAAT2 allows the expression of a bidirectional order-dependent STDP during a restricted time window.

EAA2 blockade-induced depolarization and plasticity. We investigated whether the observed plasticity was due to the transient depolarization induced by EAAT2 blockade. For this purpose, we maintained the recorded MSNs at $-80 \mathrm{mV}$ by intracellular current injection (close to MSN resting membrane

\section{Figure 1 | Corticostriatal STDP occurs within a restricted time window. (a) Scheme of the recording and stimulating sites in corticostriatal slices.} (b) STDP pairings: a single spike evoked in the recorded striatal MSN was paired with a single cortical stimulation; this pairing being repeated 100 times at $1 \mathrm{~Hz}$. $\Delta t_{\text {STDP }}$ indicates the time between pre- and postsynaptic stimulations. $\Delta t_{\text {STDP }}<0$ and $\Delta t_{\text {STDP }}>0$ refer to post-pre and pre-post pairings, respectively. (c) Example of LTP induced by 100 post-pre pairings $\left(\Delta t_{\mathrm{STDP}}=-12 \mathrm{~ms}\right.$ ). Top, EPSC strength before and after pairings. Bottom, time course of Ri (baseline: $67 \pm 0.3 \mathrm{M} \Omega$ and $50-60 \mathrm{~min}$ after pairings: $79 \pm 0.8 \mathrm{M} \Omega$; change of $18 \%$ ). EPSC traces during 10 min of baseline (1) and at $1 \mathrm{~h}$ after the STDP protocol (arrow) (2). (d) Example of LTD induced by 100 pre-post pairings ( $\Delta t_{\mathrm{STDP}}=+13 \mathrm{~ms}$; Ri, baseline: $106 \pm 0.5 \mathrm{M} \Omega ; 50-60 \mathrm{~min}$ after pairings: $116 \pm 0.5 \mathrm{M} \Omega$; change of $9 \%$ ). (e) Averaged time-course of LTP induced by 100 post-pre pairings and LTD induced by 100 pre-post pairings. (f) Bidirectional STDP occurred in a narrow time window: post-pre pairings $\left(-30<\Delta t_{\mathrm{STDP}}<0 \mathrm{~ms}\right)$ induced LTP, whereas pre-post pairings $\left(0<\Delta t_{\mathrm{STDP}}<+30 \mathrm{~ms}\right)$ induced LTD. Synaptic strength was determined 45-60 min after pairings (empty circles: individual neurons; black circle: average). The $y$ axis is discontinuous for clarity; plasticity amplitudes above the interruption are $312 \mathrm{pA}, 367 \mathrm{pA}$ and $424 \mathrm{pA}$. (g) Uncorrelated post-pre $\left(-250<\Delta t_{\text {STDP }}<-100\right)$ and pre-post $\left(+100<\Delta t_{\text {STDP }}<+250 \mathrm{~ms}\right)$ pairings induced no significant plasticity. (h) Post-pre or pre-post pairings with $\Delta t_{\text {STDP }} \sim \pm 500 \mathrm{~ms}$ induced no significant plasticity. (i) Graph summarizing STDP occurrence. Bidirectional plasticity was induced over a narrow time window $\left(-30<\Delta t_{\text {STDP }}<+30 \mathrm{~ms}\right.$ ), whereas no plasticity was observed with uncorrelated pairings $\left(-500<\Delta t_{\text {STDP }}<-30 \mathrm{~ms}\right.$ and $+30<\Delta t_{\text {STDP }}<+500 \mathrm{~ms}$ ). Insets correspond to a mean of 60 EPSCs during baseline and at $1 \mathrm{~h}$ after STDP pairings. Error bars represent the s.d. (except in f,i: s.e.m.). ${ }^{\star} P<0.05$; ${ }^{\star \star \star} P<0.001$; NS: not significant by unpaired $t$-test, two-tailed $(\mathbf{c}, \mathbf{d})$ or one sample $t$-test $(\mathbf{e}-\mathbf{i})$. 
potential) during STDP pairings, to prevent DHK-induced depolarization (Fig. 3a). In these conditions, pairings for $70<\Delta t_{\mathrm{STDP}}<+70 \mathrm{~ms}$ and $\Delta t_{\mathrm{STDP}}= \pm 200 \mathrm{~ms}$ induced LTD (77 $\pm 7 \%, P=0.0233, n=5 ; 5 / 5$ cells displayed LTD; Fig. $3 \mathrm{~b}$ ) and LTP $(186 \pm 28 \%, P=0.0382, n=5 ; 5 / 5$ cells displayed LTP;
Fig. 3c), respectively. These results are similar to those obtained in presence of DHK when neurons were not maintained at $-80 \mathrm{mV}$ (Fig. 2). Thus, the depolarization of the postsynaptic MSN induced by EAAT2 blockade does not account for the observed plasticity. a
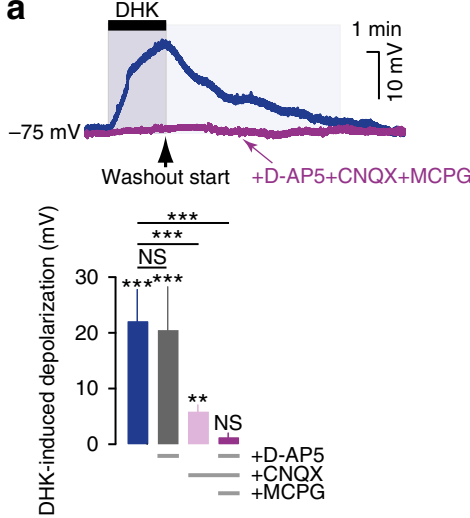

b

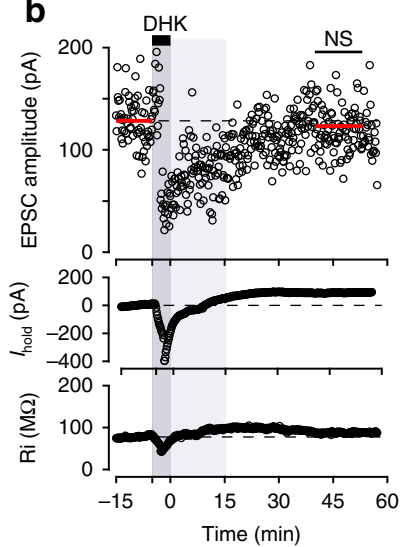

e
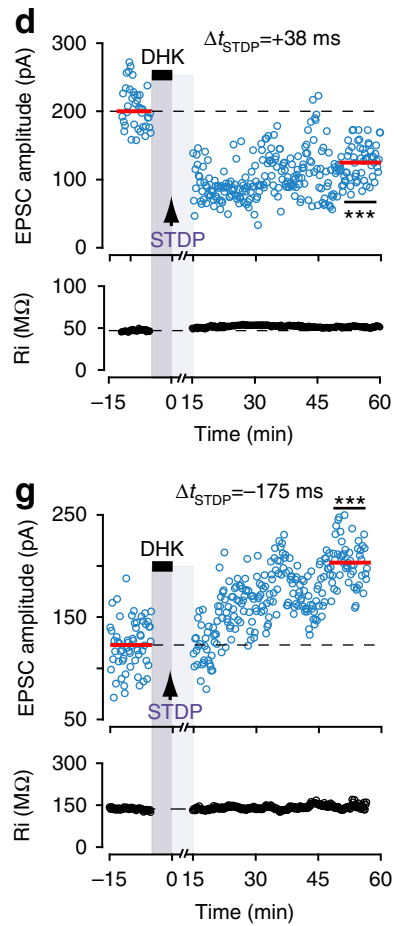

h

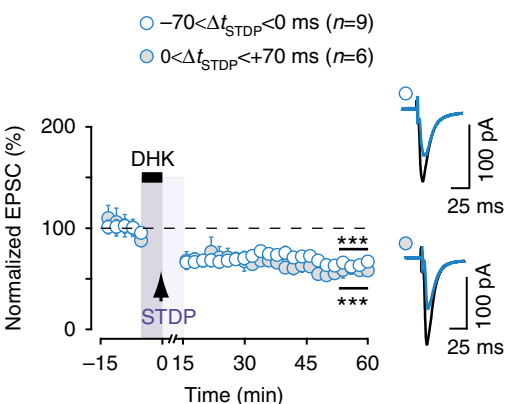

O $-250 \Delta t_{\text {STDP }}=-100 \mathrm{~ms}(n=7)$

$0+100 \Delta t_{\text {STDP }}=+250 \mathrm{~ms}(n=8)$
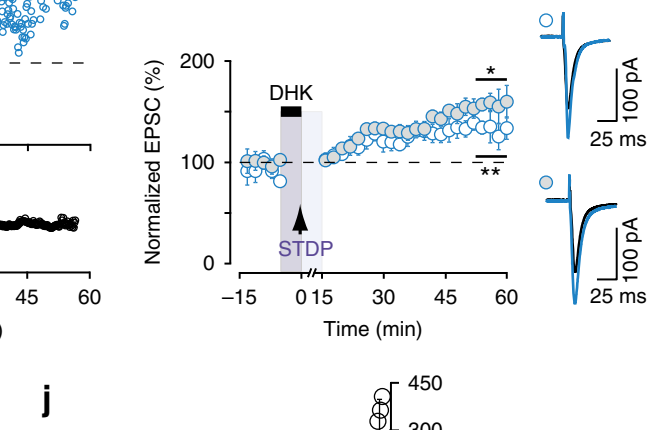

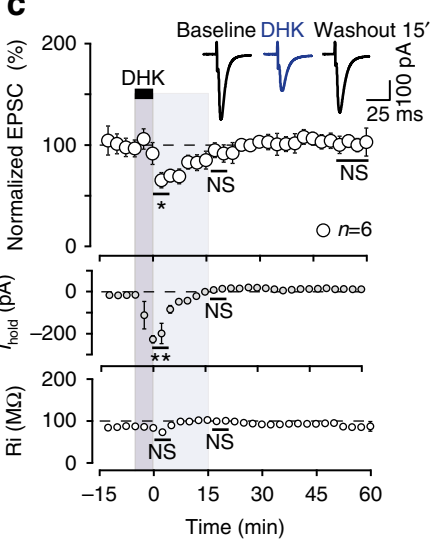

$\mathbf{f}$

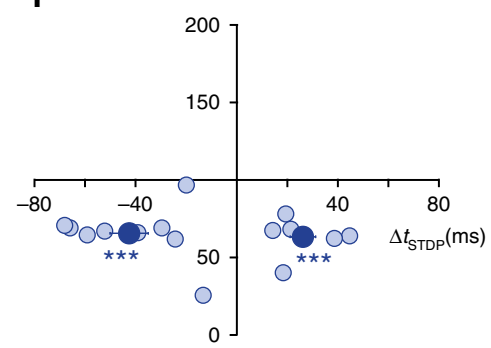

Normalized EPSC (\%)

i

$\circ \Delta t_{\mathrm{STDP}} \sim \pm 500 \mathrm{~ms}(n=7)$

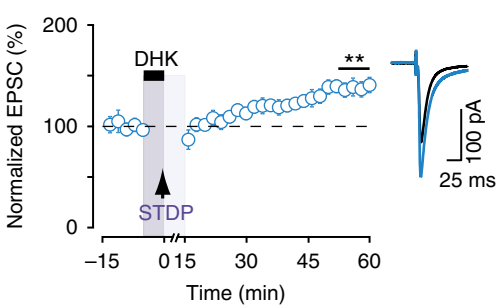

O Control

O DHK

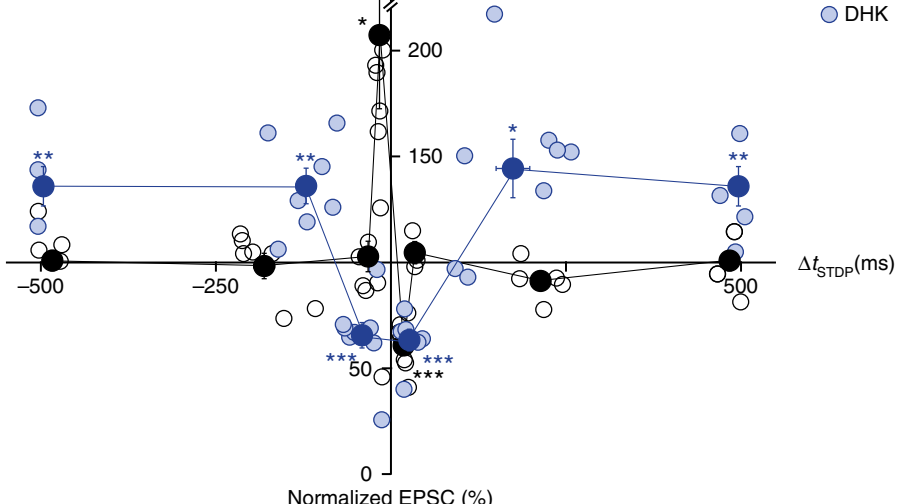

Normalized EPSC (\%) 
We then investigated whether postsynaptic depolarization alone (without DHK) during STDP pairings mimicked the effects of transient EAAT2 blockade. When MSNs were held at $-50 \mathrm{mV}$ in the absence of DHK during the STDP protocol (Fig. 3d), pairings for $-70<\Delta t_{\mathrm{STDP}}<+70 \mathrm{~ms}$ and for $\Delta t_{\mathrm{STDP}}= \pm 200 \mathrm{~ms}$ induced exclusively LTD $(65 \pm 7 \%, P=0.0029, n=7,7 / 7$ cells displayed LTD and $62 \pm 6 \%, P=0.0011, n=7,7 / 7$ cells displayed LTD, respectively; Fig. 3e,f). This result is in accordance with LTD induced with sustained depolarization in visual cortex ${ }^{35}$, and with hippocampal depolarization-induced $\mathrm{LTD}^{36}$. Thus, postsynaptic depolarization in the absence of DHK is not sufficient to reproduce the effects of transient EAAT2 blockade. Glutamate spillover is, therefore, likely to contribute to the observed plasticity.

GABA-dependent LTD under transient EAAT2 blockade. We then investigated the receptors involved in the synaptic plasticity induced under transient EAAT2 blockade. We first investigated the receptors involved in the LTD observed for pairings at $-70<\Delta t_{\mathrm{STDP}}<+70 \mathrm{~ms}$. In control conditions, corticostriatal t-LTD is mediated by $\mathrm{CB}_{1} \mathrm{R}^{16-18}$. We, therefore, first determined whether the LTD observed under EAAT2 blockade was $\mathrm{CB}_{1} \mathrm{R}$-mediated. Following the bath application of a $\mathrm{CB}_{1} \mathrm{R}$-specific antagonist (AM251;3 $3 \mathrm{M}$ ), LTD was still observed under EAAT2 blockade $(69 \pm 8 \%, P=0.0019, n=11$; 10/11 cells showed LTD; Supplementary Fig. 2a), indicating that LTD was not $\mathrm{CB}_{1} \mathrm{R}$-mediated. mGluRs and NMDARs located outside the synapse can be activated by glutamate spillover promoted by EAAT2 blockade ${ }^{15,37-40}$. We, therefore, investigated the involvement of mGluRs and NMDARs in LTD under EAAT2 blockade for pairings at $-70<\Delta t_{\mathrm{STDP}}<+70 \mathrm{~ms}$. The inhibition of type I/II mGluRs with MCPG $(500 \mu \mathrm{M})$ or of NMDARs with D-AP5 $(50 \mu \mathrm{M})$ had no effect on the establishment of LTD $(62 \pm 9 \%, P=0.0279, n=4 ; 4 / 4$ cells displayed LTD and $61 \pm 5 \%, P=0.0003, n=7 ; 7 / 7$ cells displayed LTD, respectively; Supplementary Fig. 2b). We then examined the involvement of $\mathrm{L}$ - and T-type voltage-sensitive calcium channels (VSCCs), which can be activated by DHK-induced depolarization. Under EAAT2 blockade, bath-applied mibefradil $(20 \mu \mathrm{M})$, a specific antagonist of T-type VSCCs (also blocking L-type VSCCs at concentrations above $18 \mu \mathrm{M}$ ) not only prevented LTD, but also revealed potent
LTP $(207 \pm 13 \%, \quad P=0.0002, n=7 ; 7 / 7$ cells displayed LTP; Fig. 4a). This LTP, unmasked by VSCC inhibition, was mediated by NMDARs, because it was prevented by the co-application of mibefradil and D-AP5 (84 $\pm 8 \%, P=0.0680, n=8 ; 1 / 8$ cells displayed LTP; Fig. 4a).

Given the involvement of VSCCs in the LTD observed under EAAT2 blockade, we investigated the calcium dependence of LTD at the level of the recorded MSN. To do so, we delivered intracellularly a fast calcium buffer, BAPTA, (i-BAPTA, $10 \mathrm{mM}$ ) through the patch-clamp pipette in the recorded MSN. Under EAAT2 blockade, i-BAPTA had no effect on LTD $(77 \pm 9 \%$, $P=0.0482, n=7 ; 5 / 7$ cells displayed LTD at $-70<\Delta t_{\mathrm{STDP}}<$ $+70 \mathrm{~ms}$; Fig. 4b). Thus, LTD observed under EAAT2 blockade is not dependent on postsynaptic MSN calcium. These results indicate that network effects are involved in LTD expression. They also suggest that VSCCs involved are located on neurons other than the recorded MSN and are activated during EAAT2 blockade, due to glutamate spillover-induced depolarization.

We then investigated the involvement of inhibitory networks in LTD. DHK-induced depolarization would also affect GABAergic interneurons resulting in an increased inhibitory tone ${ }^{38}$. Thus, the observed LTD might arguably arise from an increase in GABA release.

We investigated whether DHK application resulted in an increase in the inhibitory component recorded in MSNs. When MSNs were held at $-50 \mathrm{mV}$, a membrane potential for measuring mainly inhibitory transmission, we observed an outward current of $21 \pm 4 \mathrm{pA}(n=14)$ (Fig. $4 \mathrm{c})$. In the presence of $\mathrm{DHK}$, this outward current increased by $81 \%$, reaching $37 \pm 6 \mathrm{pA}$, and was inhibited by a $\mathrm{GABA}_{\mathrm{A}} \mathrm{R}$ blocker, picrotoxin $(50 \mu \mathrm{M})$, (PSC after picrotoxin: $12 \pm 1 \mathrm{pA}$; one-way repeatedmeasures ANOVA: $P<0.002$; post hoc Bonferroni-corrected pairwise comparisons: control-DHK: $P<0.01$, DHK-picrotoxin: $P<0.001)$. We tested the activation of GABAergic circuits under EAAT2 blockade directly, by making recordings on both striatal fast-spiking (FS) GABAergic interneurons and MSNs during EAAT2 blockade with DHK (Fig. 4d). In brain slices, both FS cells and MSNs are silent at rest, and DHK application led to marked depolarization in both cell types (FS cells: $+29 \pm 2 \mathrm{mV}$, $n=5$; MSNs: $+24 \pm 1 \mathrm{mV}, n=6$; Fig. 4e). Spontaneous firing activity during DHK application was observed only in FS cells $(13 \pm 7 \mathrm{~Hz}, n=5)$ whereas MSNs remained silent (Fig. 4f).

Figure 2 | EAAT2 activity gates STDP polarity and time window. (a) Current-clamp recording of MSN in the absence of cortical stimulation showing that brief DHK application ( $300 \mu \mathrm{M}$ for $5 \mathrm{~min}$ ) induced significant depolarization, indicating the presence of ambient glutamate in the slice. This depolarization was fully reversed after $15 \mathrm{~min}$ of DHK washout and was dependent on AMPAR and type-I/II mGluR, but not NMDAR. (b,c) DHK application had no effect on long-term synaptic efficacy changes estimated from $15 \mathrm{~min}$ after DHK washout (example in $\mathbf{b}$ and averaged time-course of experiments in $\mathbf{c}$ ). The brief application of DHK without the STDP protocol induced a transient decrease in EPSC amplitude and an inward shift in I holding (light gray area). Both EPSC amplitude and $I_{\text {holding }}$ had fully recovered 15 min after DHK washout. Ri remained unchanged during and after DHK application. The effects of DHK were fully reversible and, thus, compatible with the estimation of long-term synaptic efficacy changes. (d) Example of LTD induced by 100 pre-post pairings $\left(\Delta t_{\text {STDP }}=+38 \mathrm{~ms}\right)$ with a transient blockade of EAAT2 by DHK $(300 \mu \mathrm{M}$ for $5 \mathrm{~min}$, dark gray area; the light gray area indicates DHK washout). Top, EPSC strength before and after pairings. Bottom, time course of Ri (baseline, $47 \pm 0.2 \mathrm{M} \Omega ; 50-60$ min after pairings, $51 \pm 0.1 \mathrm{M} \Omega$; change of $10 \%$ ). (e) Averaged time-course of experiments with the transient blockade of EAAT2 with DHK, showing the induction of LTD for both post-pre $\left(-70<\Delta t_{\text {STDP }}<0\right.$ ms $)$ and pre-post $\left(0<\Delta t_{\mathrm{STDP}}<+70 \mathrm{~ms}\right)$ pairings. (f) LTD expression for $-70<\Delta t_{\mathrm{STDP}}<+70 \mathrm{~ms}$ with DHK. Synaptic strength was assessed $45-60 \mathrm{~min}$ after pairings (light blue circles: individual neurons; dark blue circle: average). (g) Example of LTP induced by 100 post-pre pairings $\left(\Delta t_{\mathrm{STDP}}=-175 \mathrm{~ms}\right)$ during the transient blockade of EAAT2 with DHK (Ri, baseline: $136 \pm 0.5 \mathrm{M} \Omega ; 50-60$ min after pairings: $145 \pm 1 \mathrm{M} \Omega$; change of $6 \%$ ). (h) Averaged time-course of experiments with transient EAAT2 blockade with DHK during pairings, inducing LTP for both post-pre $\left(-250<\Delta t_{\text {STDP }}<-100 \mathrm{~ms}\right)$ and pre-post $\left(+100<\Delta t_{\text {STDP }}<+250 \mathrm{~ms}\right.$ ) pairings. (i) Averaged time-course of experiments with transient EAAT2 blockade with DHK during pairings, inducing LTP for $\Delta t_{\mathrm{STDP}} \sim \pm 500 \mathrm{~ms}$. (j) Time window for long-term synaptic strength for post-pre and pre-post pairings $\left(-500<\Delta t_{\mathrm{STDP}}<+500 \mathrm{~ms}\right)$ in control conditions and in the presence of DHK. In controls, bidirectional plasticity was induced over a narrow time window $\left(-30<\Delta t_{\mathrm{STDP}}<+30 \mathrm{~ms}\right)$ and no plasticity was observed with uncorrelated pairings $\left(-500<\Delta t_{\text {STDP }}<-30 \mathrm{~ms}\right.$ and $+30<\Delta t_{\text {STDP }}<+500 \mathrm{~ms}$ ). During transient EAAT2 blockade, plasticity was observed regardless of the $\Delta t_{\text {STDP }}$ value: LTD for narrow $\Delta t_{\text {STDP }}\left(-70<\Delta t_{\text {STDP }}<+70\right)$ and LTP for a larger $\Delta t_{\text {STDP }}$ $\left(-500<\Delta t_{\mathrm{STDP}}<-70 \mathrm{~ms}\right.$ and $\left.+70<\Delta t_{\mathrm{STDP}}<+500 \mathrm{~ms}\right)$. Insets correspond to the mean of 60 EPSCs during baseline and at $1 \mathrm{~h}$ after STDP pairings. Error bars represent the s.d. (except in panel a,f,j: s.e.m.) ${ }^{\star} P<0.05$; ${ }^{\star \star} P<0.01$; ${ }^{\star \star \star} P<0.001$; NS: not significant by unpaired $t$-test, two-tailed, inside groups after one-way ANOVA; post hoc Bonferroni comparisons test (a), unpaired $t$-test, two-tailed $(\mathbf{b}, \mathbf{d}, \mathbf{g})$ or one sample $t$-test $(\mathbf{c}, \mathbf{e}, \mathbf{f}, \mathbf{h}, \mathbf{i}, \mathbf{j})$. 

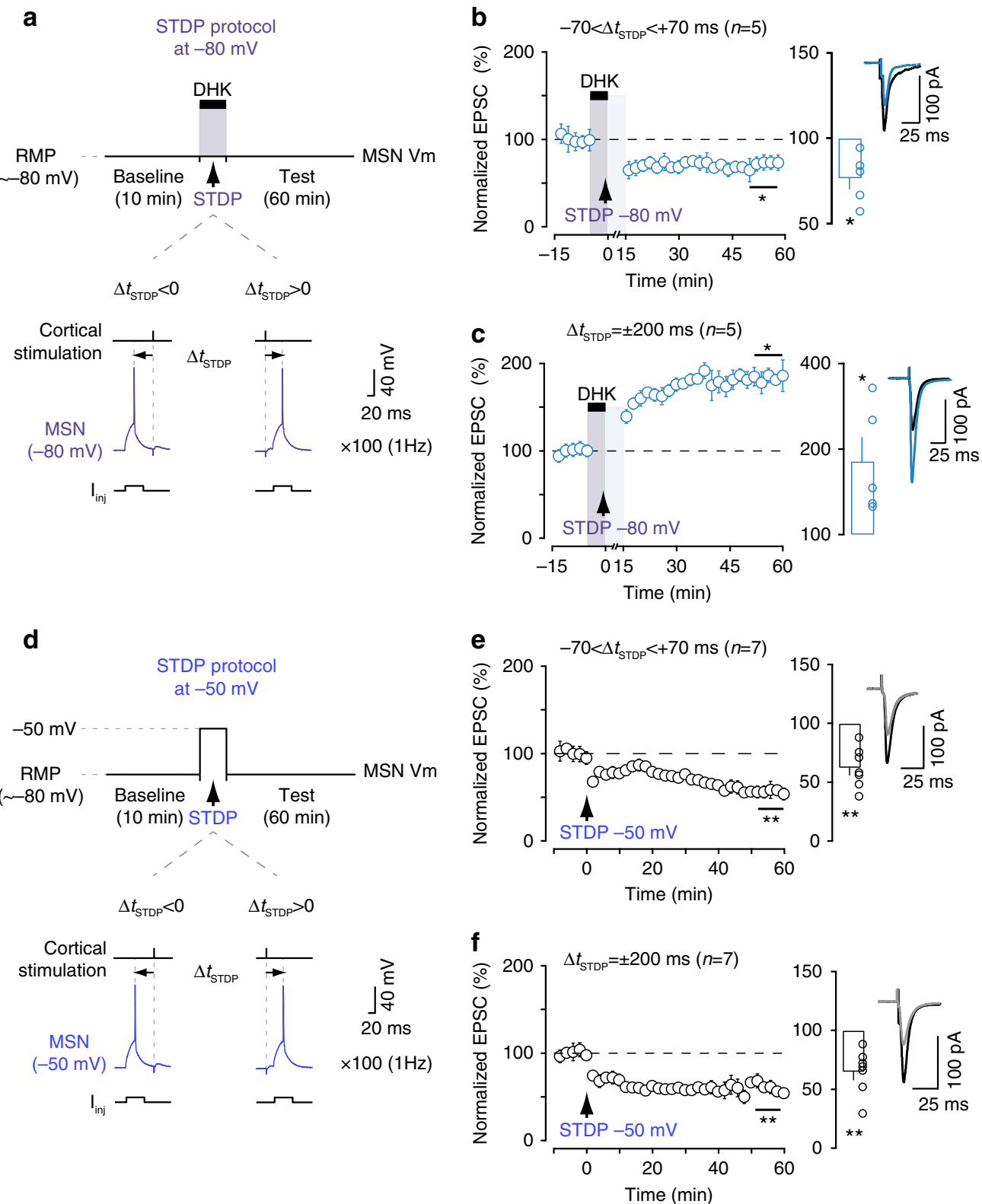

Figure 3 | Postsynaptic depolarization cannot account for plasticity under EAAT2 blockade. (a-c) Experimental protocol (a) and averaged time-course of STDP experiments $(\mathbf{b}, \mathbf{c})$ with the recorded MSN maintained at $-80 \mathrm{mV}$ by intracellular current injection during the STDP pairings. LTD and LTP were induced with pairings at $-70<\Delta t_{\mathrm{STDP}}<+70 \mathrm{~ms}(\mathbf{b})$ and $\Delta t_{\mathrm{STDP}}= \pm 200 \mathrm{~ms}$ (c), respectively. The prevention of DHK-induced depolarization did not impair the plasticity observed when MSN was depolarized. (d-f) Experimental protocol and summary of STDP experiments in which the recorded MSN was held at $-50 \mathrm{mV}(\mathbf{d})$, performed with pairings at $-70<\Delta t_{\text {STDP }}<+70 \mathrm{~ms}(\mathbf{e})$ and $\Delta t_{\text {STDP }}= \pm 200 \mathrm{~ms}$ (f), respectively; in these conditions, only LTD was observed. Insets correspond to the average of 60 EPSCs during baseline and at $1 \mathrm{~h}$ after STDP pairings. Error bars represent the s.d. (except in bar graphs: s.e.m.). ${ }^{\star} P<0.05 ;{ }^{\star \star} P<0.01$ by one sample $t$-test $(\mathbf{b}, \mathbf{c}, \mathbf{e}, \mathbf{f})$.

Cortical stimulation (of an intensity similar to that used for STDP pairings) evoked action potentials in all recorded FS cells whereas MSNs displayed subthreshold EPSPs (Fig. 4f). Thus, DHK application leads to the recruitment of GABAergic interneurons, resulting in an increase of the inhibitory weight exerted on the recorded MSN. An increase in inhibitory drive may, therefore, promote LTD.

We then bath-applied picrotoxin $(50 \mu \mathrm{M})$ to investigate the involvement of GABAergic networks in LTD. For pairings at $-70<\Delta t_{\mathrm{STDP}}<+70 \mathrm{~ms}$ under EAAT2 blockade, picrotoxin application prevented LTD, instead promoting LTP $(202 \pm 20 \%$, $P=0.0075, n=6 ; 6 / 6$ cells displayed LTP; Fig. $4 \mathrm{~g}$ ). These findings suggest that LTD was dependent on $\mathrm{GABA}_{\mathrm{A}} \mathrm{R}$ activation. Thus, an increase in inhibitory transmission, probably due to the recruitment of GABAergic interneurons under DHK treatment, is responsible for LTD. Surprisingly, the prevention of this GABAergic inhibition by picrotoxin did not result in the expected lack of plasticity. Instead, it promoted LTP. We analysed the involvement of GABAergic circuits in LTD expression further, by inhibiting GABAergic transmission during transient DHK application. Co-application of gabazine $(10 \mu \mathrm{M}$; with effects readily reversible by washout) and DHK prevented the expression of plasticity $(94 \pm 3 \%, P=0.0974, n=5 ; 1 / 5$ cells displayed LTD; Fig. 4h). Thus, GABAergic transmission during STDP pairings is determinant for LTD induction under transient EAAT2 blockade. 
O Mibefradil $(n=7)$

a $\quad-70<\Delta t_{\text {STDP }}<+70 \mathrm{~ms}$ O Mibefradil + D-AP5 $(n=8)$

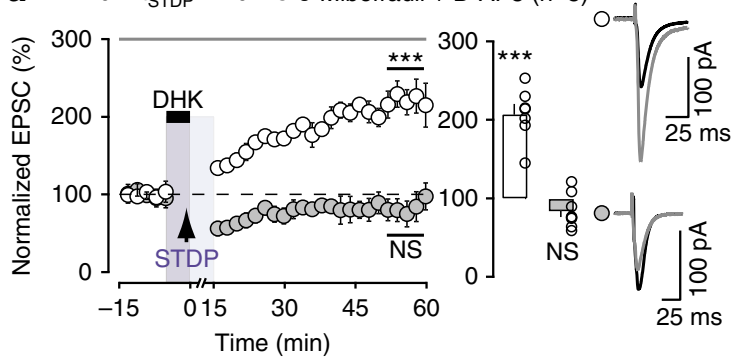

C

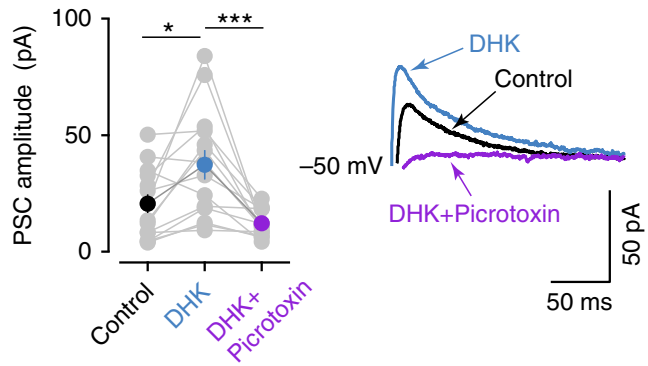

e Spontaneous activity

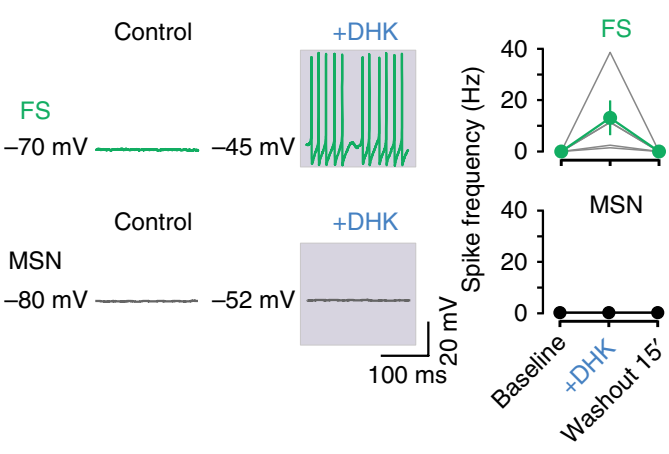

$9-70<\Delta t_{\mathrm{STDP}}<+70 \mathrm{~ms}$ Picrotoxin $(n=6)$

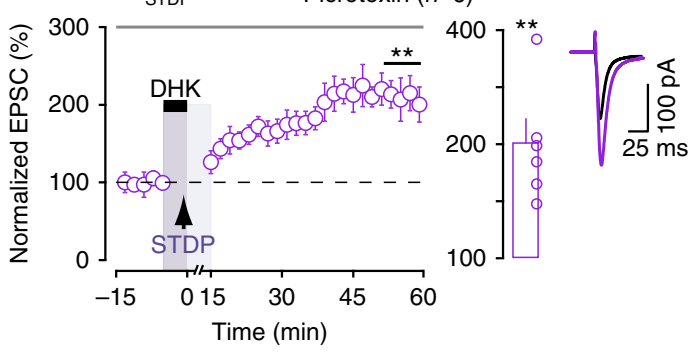

b $\quad-70<\Delta t_{\mathrm{STDP}}<+70 \mathrm{~ms} \quad$ i-BAPTA $(n=7)$
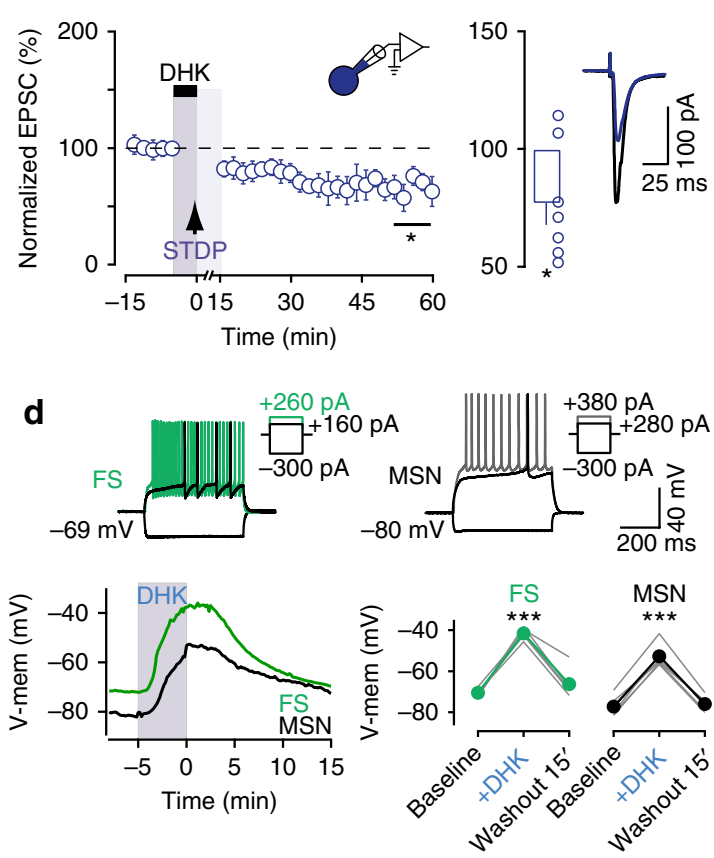

f

Triggered response
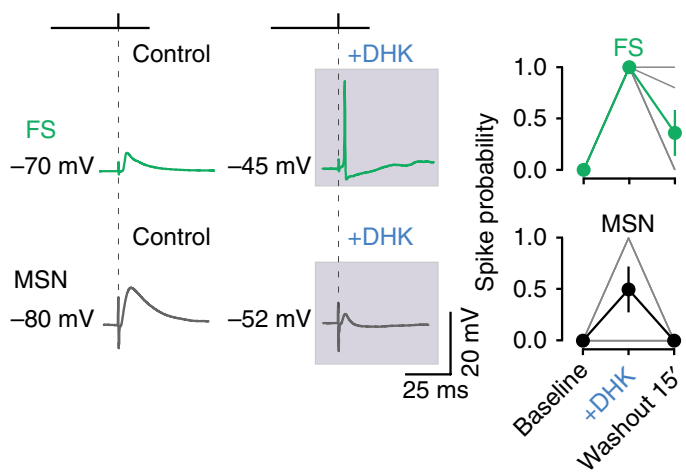

h

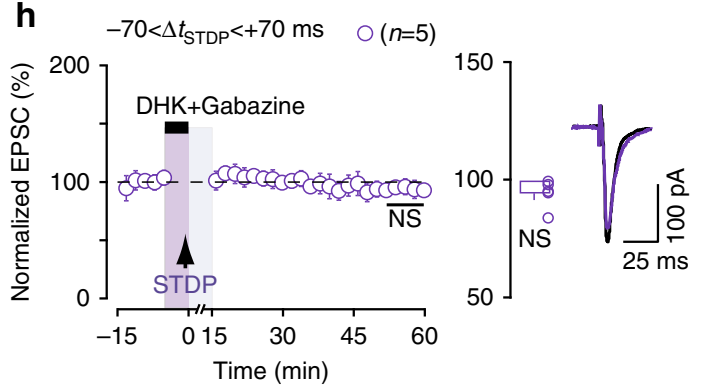

Figure 4 | GABA-dependent LTD under transient EAAT2 blockade. (a) Blocking L- and T-type VSCCs with mibefradil (20 $\mu$ M) under transient EAAT2 blockade impaired LTD and revealed potent LTP. This LTP was NMDAR-mediated, because it was prevented by the application of mibefradil (20 $\mu$ M) together with D-AP5 $(50 \mu \mathrm{M})$. (b) i-BAPTA did not impair the LTD observed under transient EAAT2 blockade. (c) Inhibitory currents recorded in MSNs held at $-50 \mathrm{mV}$ in control conditions, with DHK and with DHK + picrotoxin $(50 \mu \mathrm{M} ; n=14)$. (d) Top, characteristic voltage responses of one FS cell and one MSN to a series of 500 ms current pulses. Bottom, depolarization of FS cells and MSNs induced by DHK application. Left: example of changes in Vm before, during and after DHK application, in one FS cell and one MSN; right: mean values. (e) DHK-induced depolarization led to firing activity in FS cells but not in MSNs. (f) Under EAAT2 blockade, cortical stimulation evoked an action potential in all recorded FS cells whereas subthreshold EPSPs were observed in MSNs. (g) Picrotoxin $(50 \mu \mathrm{M})$ prevented the LTD induced by pairings at $-70<\Delta t_{\mathrm{STDP}}<+70 \mathrm{~ms}$ under EAAT2 blockade, and revealed LTP.

(h) Co-application of gabazine $(10 \mu \mathrm{M})$ with DHK during STDP pairings prevented the expression of LTD. Insets correspond to the mean of 60 EPSCs during baseline and at $1 \mathrm{~h}$ after STDP pairings. Error bars represent the s.d. $(\mathbf{a}, \mathbf{b}, \mathbf{g}, \mathbf{h})$ or the s.e.m. (c-f and bar graphs in panels $\mathbf{a}, \mathbf{b}, \mathbf{g}, \mathbf{h}) .{ }^{\star} P<0.05$; ${ }^{\star \star} P<0.01 ;{ }^{\star \star \star} P<0.001$; NS: not significant by one sample $t$-test $(\mathbf{a}, \mathbf{b}, \mathbf{g}, \mathbf{h})$, one-way repeated-measures ANOVA; post hoc Bonferroni-corrected pairwise comparisons (c) or paired t-test, two-tailed (d). 

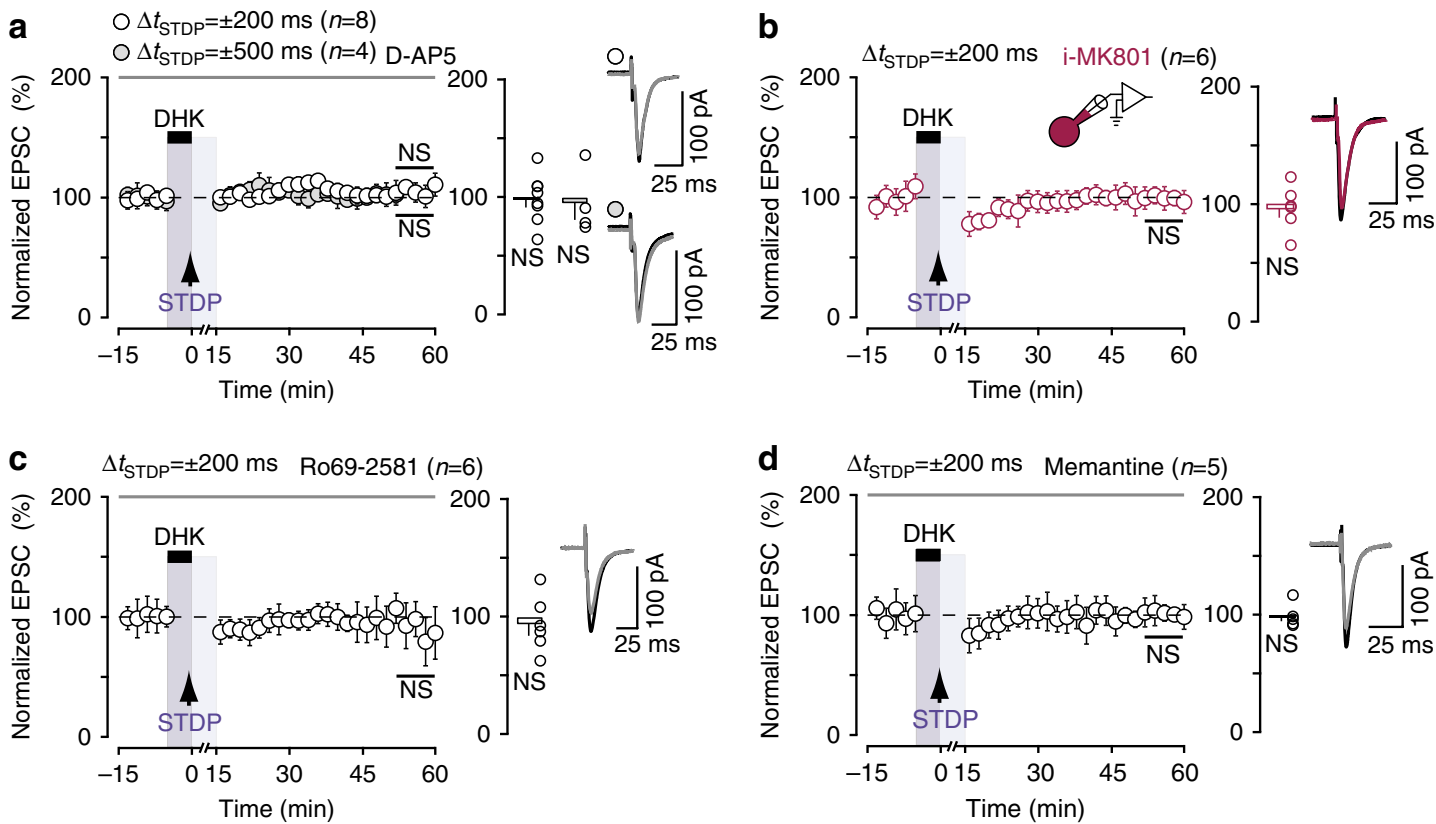

Figure 5 | Extrasynaptic GluN2B-NMDARs mediate LTP under transient EAAT2 blockade. (a) The LTP induced under EAAT2 blockade for $\Delta t_{\mathrm{STDP}}= \pm 200 \mathrm{~ms}$ and $\Delta t_{\mathrm{STDP}}= \pm 500 \mathrm{~ms}$ was mediated by NMDARs, because it was prevented by D-AP5 (50 $\mu$ M) application. (b) The LTP induced under transient EAAT2 blockade for $\Delta t_{\mathrm{STDP}}= \pm 200 \mathrm{~ms}$ was prevented by blocking postsynaptic NMDARs with i-MK801 (1 mM) applied intracellularly in the recorded MSN. (c) The inhibition of GluN2B-containing NMDARs with Ro25-6981 (10 $\mu \mathrm{M})$ prevented the induction of LTP. (d) The inhibition of extrasynaptic NMDARs with memantine $(10 \mu \mathrm{M})$ prevented LTP under transient EAAT2 blockade. Extrasynaptic GluN2B-containing NMDARs located on the postsynaptic MSN are thus required for the induction of LTP under transient EAAT2 blockade. Insets correspond to the mean of 60 EPSCs during baseline and at $1 \mathrm{~h}$ after STDP pairings. Error bars represent s.d. (except in bar graphs: s.e.m.). NS: not significant by one sample $t$-test.

The LTD observed under transient EAAT2 blockade, for pairings at $-70<\Delta t_{\mathrm{STDP}}<+70 \mathrm{~ms}$, is, thus, dependent on the activation of VSCCs, probably located on striatal GABAergic interneurons. The blockade of GABAergic transmission revealed potent LTP, similar to that observed for uncorrelated pairings $\left(-500<\Delta t_{\text {STDP }}<-70 \mathrm{~ms}\right.$ and $+70<\Delta t_{\text {STDP }}<+500 \mathrm{~ms}$ ). Thus, an impairment of EAAT2 function leads to LTP over the entire range of $\Delta t_{\mathrm{STDP}}$, with the exception of a narrow time window $\left(-70<\Delta t_{\mathrm{STDP}}<+70 \mathrm{~ms}\right)$, during which GABAergic microcircuits take over LTP and impose LTD.

Extrasynaptic GluN2B-NMDARs mediate LTP. We then investigated the mechanism underlying the LTP observed under transient EAAT2 blockade, for pairings at $-500<\Delta t_{\mathrm{STDP}}<-70 \mathrm{~ms}$ and $+70<\Delta t_{\text {STDP }}<+500 \mathrm{~ms}$. For both $\Delta t_{\text {STDP }}= \pm 200 \mathrm{~ms}$ and $\Delta t_{\text {STDP }}= \pm 500 \mathrm{~ms}$, LTP was mediated by NMDAR, as it was prevented by $\mathrm{D}-\mathrm{AP} 5(50 \mu \mathrm{M} ; 98 \pm 7 \%, P=0.8330, n=8 ; 1 / 8$ cells displayed LTP and $95 \pm 14 \%, P=0.7306, n=4 ; 1 / 4$ cells displayed LTP, respectively; Fig. 5a). Glutamate spillover induced by EAAT2 blockade has been reported to mediate crosstalk between neighboring neurons via NMDARs ${ }^{15,40}$. We therefore investigated whether the observed LTP was dependent on the recruitment of NMDARs expressed on neighboring cells or solely on the NMDARs located on the postsynaptic MSN subjected to pairings. We used MK801, a use-dependent blocker of NMDARs, which we delivered intracellularly to the postsynaptic MSN used for recording via the patch-clamp pipette (i-MK801; $1 \mathrm{mM}$ ). i-MK801 prevented LTP ( $97 \pm 8 \%, P=0.6777, n=6 ; 1 / 6$ cells displayed LTP; Fig. 5b). The NMDARs required for LTP were, therefore, located on the postsynaptic recorded MSN, and not on neighboring cells. We then aimed at identifying further the NMDARs involved in the LTP observed under transient EAAT2 blockade. Glutamate spillover activates high-affinity extrasynaptic NMDARs ${ }^{14,15,39,40}$, which are enriched in the GluN2B subunit ${ }^{41}$. We thus explored the involvement of GluN2B-containing NMDARs in LTP with Ro25-6981, a selective non-competitive antagonist of the GluN2B subunit. Ro25-6981 treatment $(10 \mu \mathrm{M})$ prevented long-term plasticity $(93 \pm 10 \%, P=0.5320, n=6 ; 1 / 6$ cells displayed LTP; Fig. 5c), demonstrating the involvement of GluN2B-containing NMDARs in LTP expression under EAAT2 blockade.

The GluN2B subunit is predominantly expressed at extrasynaptic NMDARs but it has also been identified in synaptic NMDARs ${ }^{41}$. We applied memantine $(10 \mu \mathrm{M})$, a low-affinity uncompetitive NMDAR antagonist that acts as an open-channel blocker with a fast off-rate (see Methods section). Memantine preferentially blocks extrasynaptic NMDARs, without affecting synaptic transmission. Indeed, memantine blocks with a greater extend extrasynaptic NMDARs that are activated due to a low but prolonged elevation of glutamate concentration. By contrast, memantine is relatively inefficient to block NMDARs in the presence of higher synaptic concentrations of glutamate over periods of a few milliseconds, and thus does not interfere with synaptic activity ${ }^{42}$. For STDP during EAAT2 blockade, memantine treatment prevented LTP, as no significant plasticity was observed $(99 \pm 5 \%, P=0.8302, n=5 ; 1 / 5$ cells displayed LTP; Fig. 5d). Extrasynaptic GluN2B-containing NMDARs located on the postsynaptic recorded striatal MSN are thus required for LTP induction under EAAT2 blockade.

We previously showed that corticostriatal t-LTP is dependent on postsynaptic NMDARs ${ }^{31}$ and, more precisely, that the balance between GluN2A- and GluN2B-containing NMDARs shapes $\Delta t_{\text {STDP }}{ }^{43}$. We further investigated whether extrasynaptic NMDARs were required for t-LTP expression in control conditions, as observed for as for LTP observed under EAAT2 blockade. For this purpose, we performed STDP experiments with post-pre pairings at $-30<\Delta t_{\mathrm{STDP}}<0 \mathrm{~ms}$ (similar to the experiments in Fig. 1c,e), in presence of memantine $(10 \mu \mathrm{M})$; LTP was still observed $(222 \pm 44 \%, \quad P=0.0271, \quad n=8 ; 7 / 8$ cells 
a

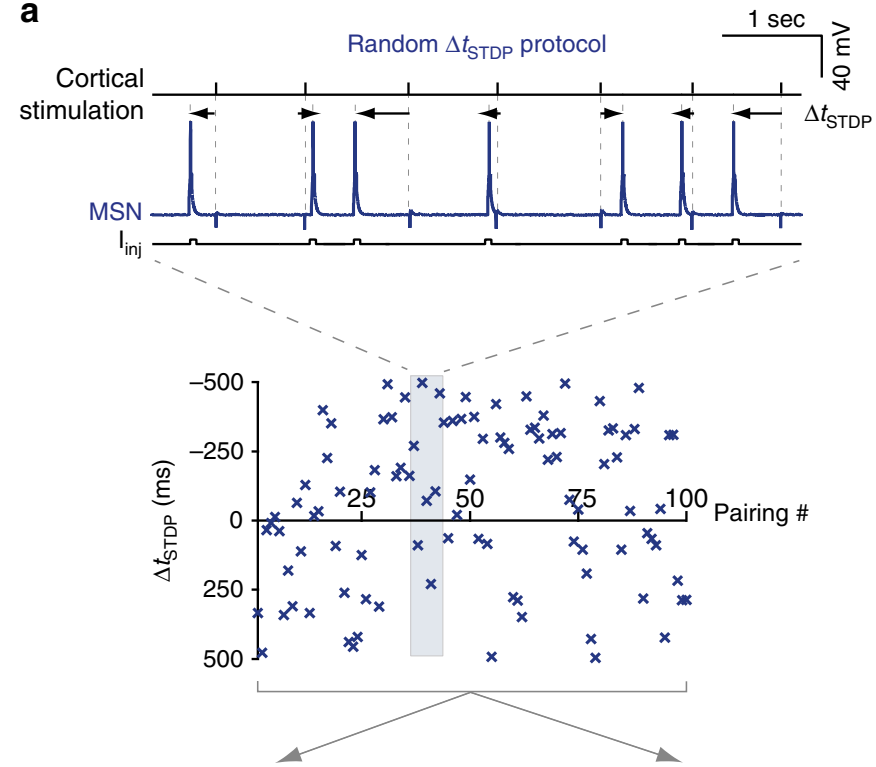

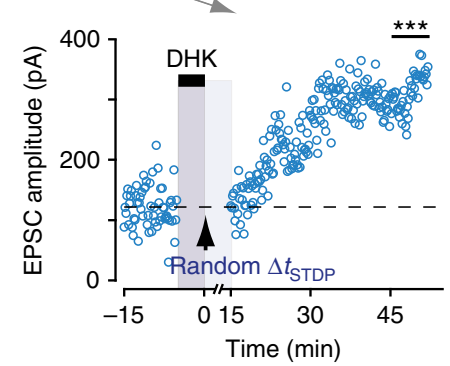
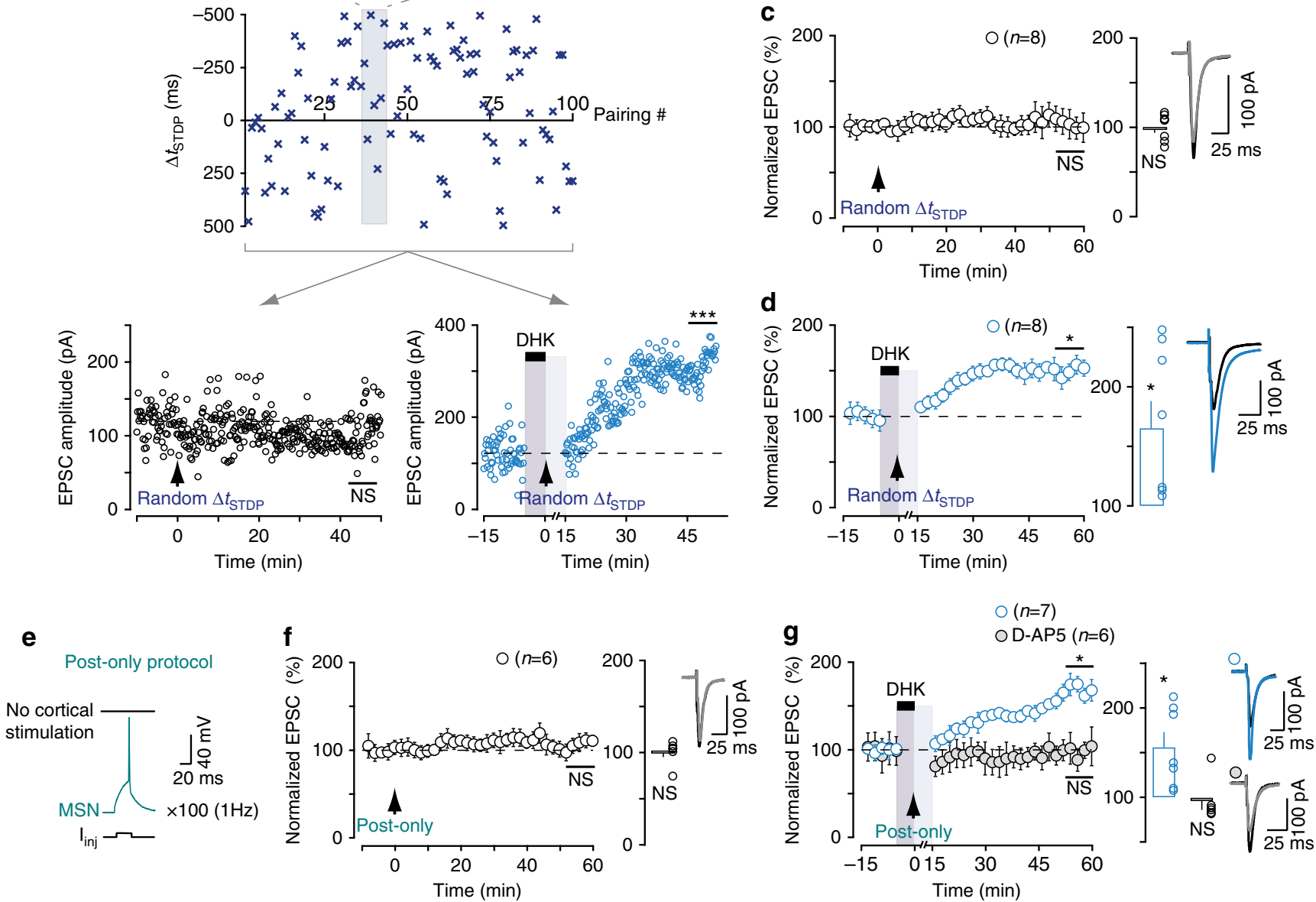

Figure 6 | LTP under transient EAAT2 blockade is not dependent on paired activity. (a) Example of one random $\Delta t_{\text {STDP }}$ pairing in control conditions and of one such pairing under transient EAAT2 blockade. Top, scatter plot of a single random $\Delta t_{\text {STDP }}$ pattern (comprising 100 consecutive random $\Delta t_{\text {STDP }}$ pairings between -500 and $+500 \mathrm{~ms}$ ) together with the CC traces of 7 successive random pairings. Bottom, examples of experiments performed in two separate MSNs, showing that the same random $\Delta t_{\text {STDP }}$ pattern failed to induce plasticity in control conditions, whereas LTP was observed under transient EAAT2 blockade. (b) Histogram of the $\Delta t_{\text {STDP }}$ from the $n=8$ random protocols, showing a uniform distribution. (b-d) Eight random $\Delta t_{\text {STDP }}$ patterns were generated and each was applied to two MSNs, one in control conditions (c) and the other under EAAT2 blockade (d). In summary, random $\Delta t_{\text {STDP }}$ patterns failed to induce plasticity in control cells, but resulted in LTP under transient EAAT2 blockade. Thus, under transient EAAT2 blockade, plasticity is not dependent on the timing and order of the paired activity. (e) Experimental design depicting a cell conditioning protocol consisting of a postsynaptic spike without paired presynaptic stimulation, repeated 100 times at $1 \mathrm{~Hz} ;(\mathbf{f})$ This protocol did not induce plasticity in control conditions. (g) Postsynaptic suprathreshold activity is sufficient to induce potent LTP under transient EAAT2 blockade. This LTP was mediated by NMDARSs, as it was prevented by D-AP5 $(50 \mu \mathrm{M})$. Insets correspond to the mean of 60 EPSCs during baseline and at $1 \mathrm{~h}$ after STDP pairings. Error bars represent the s.d. (except in bar graphs: s.e.m.). ${ }^{\star} P<0.05 ;{ }^{\star \star \star} P<0.001$; NS: not significant by unpaired $t$-test, two-tailed (a) or one sample $t$-test $(\mathbf{c}, \mathbf{d}, \mathbf{f}, \mathbf{g})$.

displayed LTP; Supplementary Fig. 3). Thus, in control conditions, extrasynaptic NMDARs are not required for t-LTP expression. This finding is consistent with the observation that, compared with t-LTP in control conditions, the LTP induced for uncorrelated pairings under transient EAAT2 blockade involves distinct signalling pathways.

LTP under transient EAAT2 blockade is not time or orderdependent. Under transient EAAT2 blockade, plasticity was observed even for highly uncorrelated pairings (up to $\Delta t_{\mathrm{STDP}}= \pm 500 \mathrm{~ms}$; Fig. $2 \mathrm{~g}$ ). This suggests that the induction of plasticity is not dependent on the timing or order of pre- and postsynaptic activity. Timing, order and paired activity are the cardinal features of STDP ${ }^{11}$. We, therefore, investigated whether the plasticity observed under transient EAAT2 blockade nevertheless followed STDP rules. We designed STDP protocols with each of $100 \Delta t_{\text {STDP }}$ pairings chosen randomly between -500 and $+500 \mathrm{~ms}$ from a close-to-uniform distribution 
(see Methods section; Fig. 6). Each of the random pairing protocols $(n=8)$ was applied both to a MSN recorded in control conditions and to a MSN subjected to transient EAAT2 blockade. An example is shown in Fig. 6a, with two MSNs (one in control conditions and the other under transient EAAT2 blockade) subje- cted to the same random pairing template. A single random $\Delta t_{\text {STDP }}$ pattern (taken from the eight different randomly generated $\Delta t_{\text {STDP }}$ patterns) did not trigger plasticity in the MSN in control conditions (the mean baseline EPSC amplitude, $119 \pm 3 \mathrm{pA}$, was not significantly different from the $120 \pm 5 \mathrm{pA}$ a

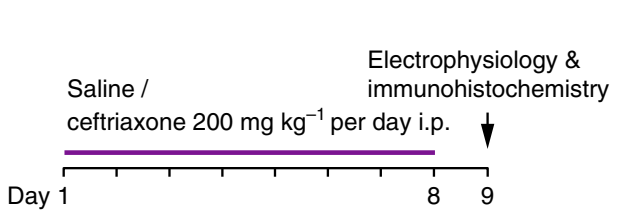

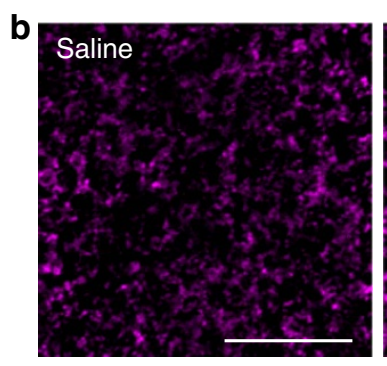
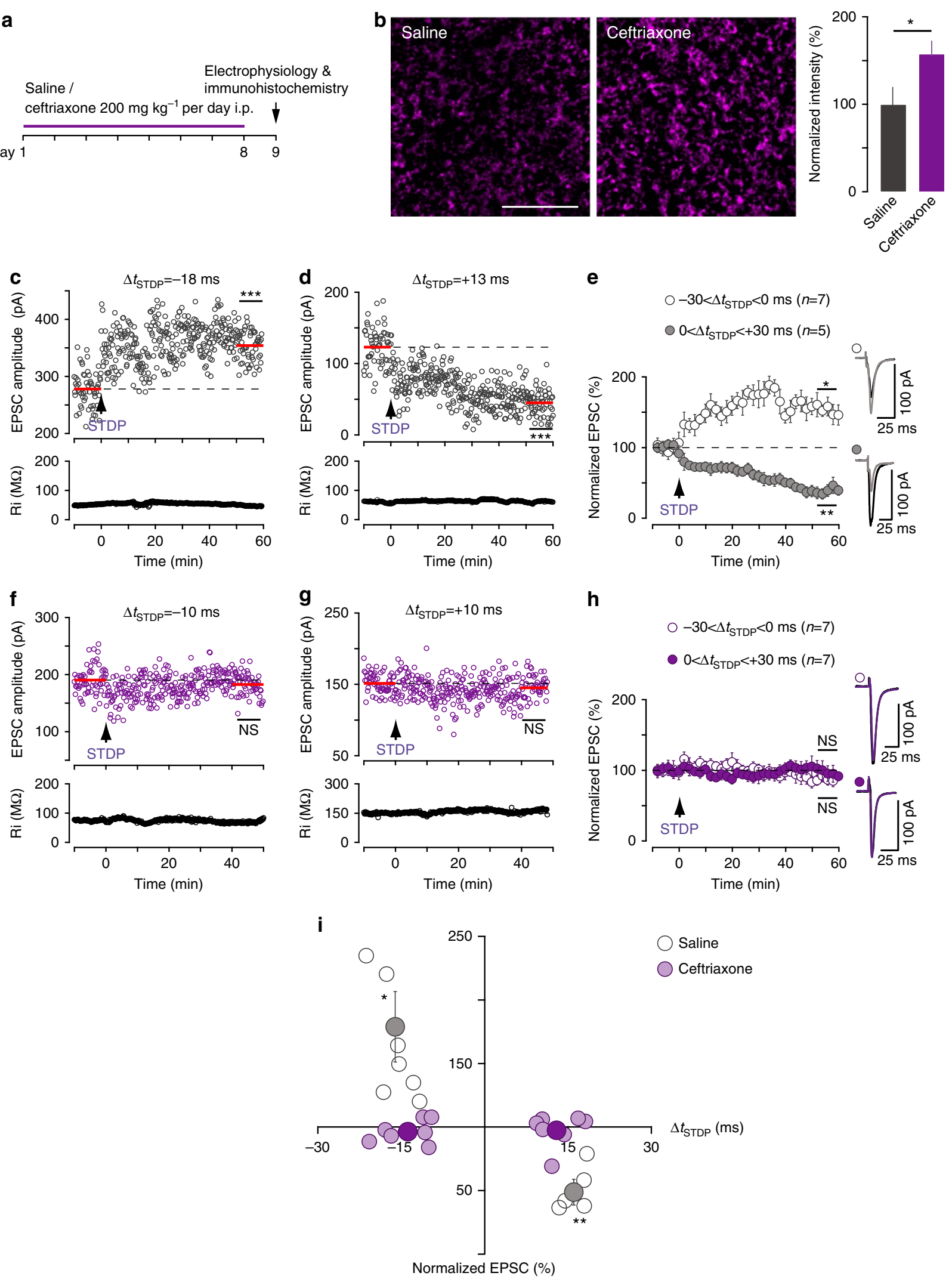

h

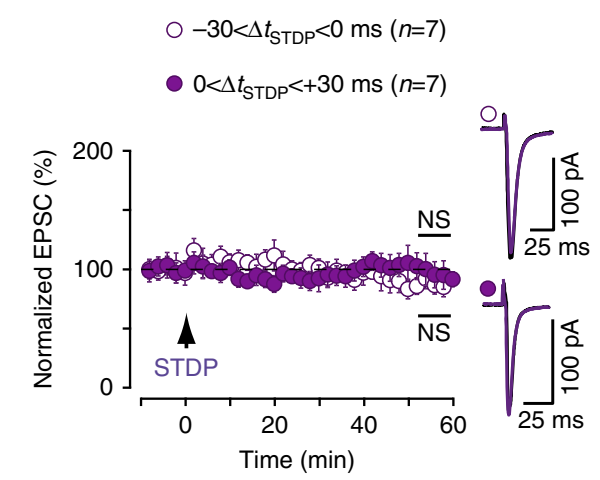


$1 \mathrm{~h}$ after pairings), but it did induce LTP in the MSN subjected to transient EAAT2 blockade (the mean baseline EPSC amplitude, $122 \pm 4 \mathrm{pA}$, increased by $52 \%$, to $307 \pm 4 \mathrm{pA}, 1 \mathrm{~h}$ after pairings). The histogram of the $\Delta t_{\text {STDP }}$ random pairings $(n=8)$ in Fig. $6 \mathrm{~b}$ illustrates that pairings were randomly distributed in a uniform manner. The application of the eight different randomly generated $\Delta t_{\text {STDP }}$ patterns resulted in no significant plasticity in control conditions $(99 \pm 5 \%, P=0.8429, n=8 ; 2 / 8$ cells displayed LTP; Fig. 6c), whereas these patterns induced LTP under transient EAAT2 blockade $(165 \pm 22 \%, P=0.0226, n=8$; 7/8 cells displayed LTP; Fig. $6 \mathrm{~d}$ ). Thus, plasticity under transient EAAT2 blockade does not depend on the timing or order of the paired activity on either side of the synapse and does not, therefore, meet the criteria for STDP.

LTP under transient EAAT2 blockade does not require paired activity. The timing and order of pairings are crucial for STDP, but were not critical for the expression of plasticity under EAAT2 blockade. We investigated whether paired activity was required to induce plasticity under EAAT2 blockade, by determining whether unpaired activity consisting in postsynaptic spiking (a single postsynaptic action potential repeated 100 times at $1 \mathrm{~Hz}$ ) without presynaptic stimulation could trigger long-term plasticity (Fig. 6e). In control conditions, this unpaired activity did not induce plasticity $(101 \pm 5 \%, P=0.9074, n=6 ; 1 / 6$ cells displayed LTP; Fig. 6f). By contrast, under transient EAAT2 blockade, this unpaired activity was sufficient to trigger LTP (156 $\pm 17 \%$, $P=0.0152, n=7 ; 6 / 7$ cells displayed LTP; Fig. $6 \mathrm{~g})$. This LTP was prevented by D-AP5 $(50 \mu \mathrm{M})$ and was therefore NMDARmediated ( $96 \pm 10 \%, P=0.6693, n=6 ; 1 / 6$ cells displayed LTP; Fig. 6g).

Finally, we investigated whether postsynaptic suprathreshold activity was required to induce plasticity under transient EAAT2 blockade. To do so, we induced subthreshold depolarization (repeated 100 times at $1 \mathrm{~Hz}$ without cortical stimulation) in the recorded MSN (Supplementary Fig. 4a). This subthreshold unpaired postsynaptic stimulation was not sufficient to trigger significant plasticity when the average of all experiments performed in these conditions was considered: $118 \pm 10 \%(P=0.1213, n=6$; Supplementary Fig. 4b). However, four of the six recorded MSNs displayed significant LTP (see scatter plot in Supplementary Fig. 4b). The postsynaptic spike therefore seems to be required for the induction of potent NMDAR-mediated LTP under transient EAAT2 blockade.

Correct functioning of EAAT2 is, therefore, required for STDP expression. A cardinal feature for STDP is that it relies on the precise time-correlation between the activities on either side of the synapse. Plasticity under transient EAAT2 blockade therefore does not meet the criteria for STDP.

EAAT2 overexpression prevents striatal STDP. To estimate to what extent EAAT2 controls STDP expression, we next questioned if an overexpression of EAAT2 would have an impact on STDP. We used ceftriaxone, a beta-lactam antibiotic that increases EAAT2 levels and activity ${ }^{44}$. Indeed, immunohistochemistry showed that eight days of daily i.p. ceftriaxone $\left(200 \mathrm{mg} \mathrm{kg}^{-1}\right)$ injections in rats (Fig. 7a) significantly increased $(P=0.0420)$ EAAT2 levels in the striatum (Fig. $7 \mathrm{~b})$. The control group consisted of rats receiving a daily injection of an equal volume of saline for 8 days. We observed no significant difference between saline- and ceftriaxone-injected rats for passive and active membrane properties of MSNs (RMP, Ri, rheobase, intensity-frequency relationship), transmission and short-term plasticity (Supplementary Fig. 5a-i) or NMDAR-mediated EPSCs (Supplementary Fig. 5j-1). We verified that similar STDP was observed in saline-injected and control rats. The examples in Fig. $7 \mathrm{c}, \mathrm{d}$ show that post-pre pairings at $\Delta t_{\mathrm{STDP}}=-18 \mathrm{~ms}$ induced LTP (the mean baseline EPSC amplitude was $278 \pm 4 \mathrm{pA}$ before pairings and had increased by $27 \%$, to $354 \pm 3$ pA, $1 \mathrm{~h}$ after pairings; Fig. 7c) whereas pre-post pairings at $\Delta t_{\mathrm{STDP}}=+13 \mathrm{~ms}$ induced LTD (the mean baseline EPSC amplitude was $123 \pm 4 \mathrm{pA}$ before pairings and had decreased by $63 \%$, to $45 \pm 2 \mathrm{pA}, 1 \mathrm{~h}$ after pairings; Fig. $7 \mathrm{~d}$ ). In summary, salineinjected rats displayed bidirectional STDP similar to that observed in control rats: post-pre pairings induced LTP $(179 \pm 28 \%, P=0.0295, n=7 ; 7 / 7$ cells displayed LTP) and pre-post pairings triggered LTD $(51 \pm 8 \%, P=0.0036, n=5 ; 5 / 5$ cells displayed LTD; Fig. 7e,i). In ceftriaxone-treated rats, canonical pairings were unable to induce STDP. Indeed, as exemplified in Fig. $7 \mathrm{f}$, post-pre pairings at $\Delta t_{\mathrm{STDP}}=-10 \mathrm{~ms}$ failed to induce plasticity: no significant difference was observed before and after pairings $(190 \pm 3 \mathrm{pA}$ and $182 \pm 3 \mathrm{pA}$, respectively). Similarly, an absence of plasticity was observed for pre-post pairings at $\Delta t_{\mathrm{STDP}}=+10 \mathrm{~ms}$ because there was no significant difference before and after pairings $(151 \pm 2 \mathrm{pA}$ and $148 \pm 3$ pA, respectively; Fig. $7 \mathrm{~g}$ ). In summary, MSNs recorded from ceftriaxone-treated rats displayed no STDP as both post-pre $\left(-30<\Delta t_{\text {STDP }}<0 \mathrm{~ms}\right)$ and pre-post $\left(0<\Delta t_{\text {STDP }}<+30 \mathrm{~ms}\right)$ pairings failed to induce significant plasticity $(96 \pm 3 \%$, $P=0.3286, n=7,0 / 7$ cells displayed LTP and $97 \pm 5 \%$, $P=0.6279, \quad n=7, \quad 1 / 7$ cells displayed LTD, respectively; Fig. 7h,i). In conclusion, EAAT2 overexpression impaired the

Figure 7 | EAAT2 overexpression by ceftriaxone treatment impairs STDP. (a) Experimental design: ceftriaxone (or saline) was daily injected for 8 days; electrophysiology and immunohistochemistry experiments were performed $24 \mathrm{~h}$ after the last injection. (b) Immunohistochemistry revealed an increase of EAAT2-positive puncta in striatal slices from ceftriaxone-injected rats than in slices from saline-injected rats. Scale bar: $10 \mu \mathrm{m}$. (c) Example of LTP induced by 100 post-pre pairings recorded in a saline-injected rat $\left(\Delta t_{S T D P}=-18 \mathrm{~ms}\right)$. Top, EPSC strength before and after pairings. Bottom, time course of Ri (baseline: $50 \pm 0.2 \mathrm{M} \Omega ; 50-60 \mathrm{~min}$ after pairings: $48 \pm 0.2 \mathrm{M} \Omega$; change of $-5 \%$ ). (d) Example of LTD induced by 100 pre-post pairings recorded in a saline-injected rat $\left(\Delta t_{\mathrm{STDP}}=+13 \mathrm{~ms}\right.$; Ri, baseline: $60 \pm 0.3 \mathrm{M} \Omega ; 50-60 \mathrm{~min}$ after pairings: $61 \pm 0.4 \mathrm{M} \Omega$; change of $\left.0.4 \%\right)$. (e) Averaged time-course of experiments performed in saline-injected rats, showing bidirectional STDP: LTP was induced for post-pre $\left(-30<\Delta t_{\text {STDP }}<0\right.$ ms $)$ and LTD for pre-post $\left(0<\Delta t_{\text {STDP }}<+30 \mathrm{~ms}\right)$ pairings. (f) Example of the lack of plasticity observed with 100 post-pre pairings $\left(\Delta t_{\mathrm{STDP}}=-10 \mathrm{~ms}\right)$ recorded from a ceftriaxonetreated rat. Top, EPSC strength was not significantly different before and after pairings. Bottom, time course of Ri (baseline: $75 \pm 0.3 \mathrm{M} \Omega ; 40-50 \mathrm{~min}$ after pairings: $69 \pm 0.5 \mathrm{M} \Omega$; change of $-8 \%$ ). (g) Example of the absence of plasticity observed with 100 pre-post pairings $\left(\Delta t_{\text {STDP }}=+10 \mathrm{~ms}\right)$ from a ceftriaxone-treated rat. EPSC strength did not differ significantly before and after pairings (Ri, baseline: $149 \pm 0.6 \mathrm{M} \Omega ; 40-50$ min after pairings $163 \pm 10 \mathrm{M} \Omega$; change of $10 \%$ ). (h) Averaged time course of experiments performed on ceftriaxone-treated rats, showing an absence of STDP for both postpre and pre-post pairings. (i) Time window for long-term synaptic strength for post-pre and pre-post pairings $\left(-30<\Delta t_{\mathrm{STDP}}<+30 \mathrm{~ms}\right)$ in saline- and ceftriaxone-treated rats. Synaptic strength was assessed 45-60 min after pairings (empty and pink circles: individual neurons; gray or purple circles: average). Bidirectional plasticity was induced in saline-injected rats, whereas no plasticity was observed in ceftriaxone-treated rats. Insets correspond to the average of 60 EPSCs during baseline and at $1 \mathrm{~h}$ after STDP pairings. Error bars represent the s.d. (except in panel b,i: s.e.m.). ${ }^{\star} P<0.05 ;{ }^{\star \star} P<0.01$;

${ }^{\star \star \star} P<0.001$; NS: not significant by unpaired $t$-test, two-tailed $(\mathbf{b}-\mathbf{d}, \mathbf{f}, \mathbf{g})$ or one sample $t$-test $(\mathbf{e}, \mathbf{h}, \mathbf{i})$. 
a

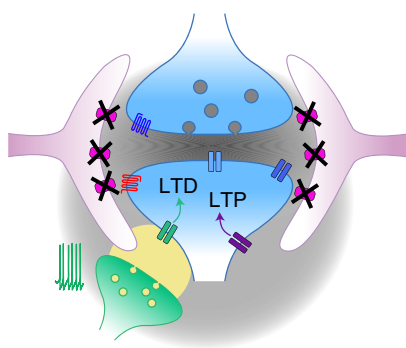

Non-Hebbian plasticity (no STDP)

Timing-independent LTP

GABA-dependent LTD b EAAT2 normal expression

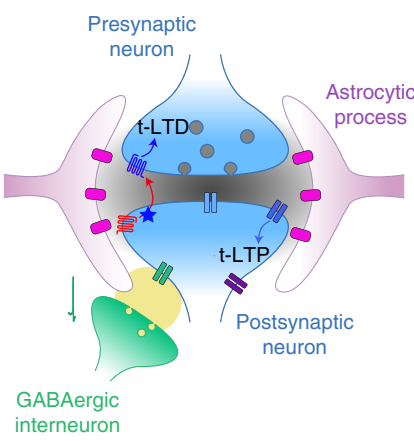

Bidirectional Hebbian STDP

Timing-dependent LTP and LTD

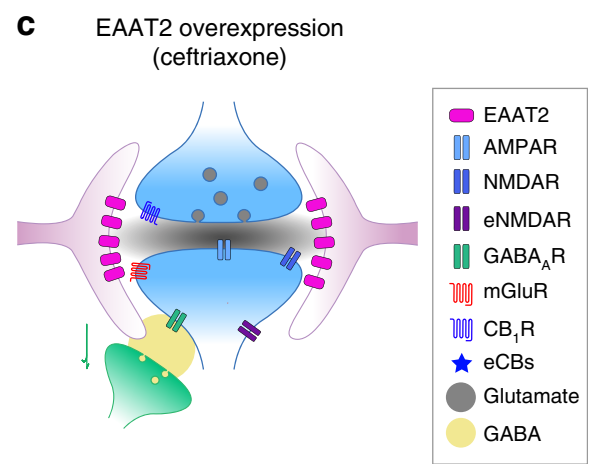

No STDP

Figure 8 | Impact of astrocytes, via EAAT2, on Hebbian plasticity. Schematic representation of the role of astrocytes, via EAAT2, on Hebbian plasticity in the striatum. (a) Transient EAAT2 blockade prevents the expression of STDP, instead favoring non-Hebbian plasticity (timing-independent LTP). This LTP is mediated by extrasynaptic NMDAR and LTD is dependent on the activation of striatal GABAergic microcircuits. In these conditions, unpaired activity is sufficient to induce LTP. (b) The physiological expression and activity of EAAT2 allows the emergence of Hebbian plasticity (bidirectional STDP). Depending on the order of pre- and postsynaptic activity, NMDAR-mediated t-LTP or endocannabinoid-mediated t-LTD is induced. (c) EAAT2 overexpression by limiting glutamate spillover prevents STDP expression. Thus, the efficiency of glutamate uptake, most through astrocytic EAAT2, gates the expression of Hebbian synaptic plasticity in the striatum.

detection of correlated activity and precluded the occurrence of a bidirectional STDP (Fig. 7i).

\section{Discussion}

Identifying the conditions required for the expression of Hebbian plasticity, such as STDP, is essential for a better understanding of the mechanisms underlying learning and memory. Our findings demonstrate that astrocytes play a key role in the establishment of STDP, through EAAT2-mediated glutamate uptake. Indeed, EAAT2 allows translating precise pre- and postsynaptic activity into a salient time-coded message. This is a key requirement for STDP, the main characteristic of which is a high degree of sensitivity to timing ${ }^{19,20}$, a feature that was erased by the transient blockade of EAAT2. Under this blockade, STDP was replaced by a non-Hebbian form of plasticity that was not dependent on the timing or order of the activities on either side of the synapse and was even observed in cases of unpaired activity. By contrast, EAAT2 overexpression impaired the detection of correlated pre- and postsynaptic activity by MSNs, resulting in an absence of plasticity. Our results show that astrocytes gate the conversion from non-Hebbian to Hebbian plasticity via EAAT2, leading to the emergence of STDP (Fig. 8).

Astrocytes actively control various synaptic functions and, therefore, play a key role in the modulation of neuronal activity $11,12,45,46$. Control of neuronal computation by astrocytes is via the release and uptake of transmitters, such as glutamate. Glutamate release by astrocytes plays an important role in STDP at L4-L2/3 neocortical synapses, by controlling t-LTD through the activation of astrocytic $C_{1} \mathrm{R}^{47}$. By contrast, the involvement of astrocytic glutamate uptake in a time-coding paradigm, such as STDP, has never been investigated. Previous reports indicate that rate-coded plasticity, induced by low- or high-frequency stimulation (LFS and HFS) or theta-burst stimulation (TBS), is sensitive to changes in astrocytic glutamate uptake ${ }^{48-53}$. In addition, neuronal EAAT3 regulates the balance between TBSLTP and LFS-LTD ${ }^{54}$ and cerebellar LTD is dependent on the patterned expression of neuronal EAAT4 on Purkinje cells ${ }^{55}$. This study is, to our knowledge, the first to assess the involvement of astrocytic glutamate uptake in the expression of time-coded plasticity. STDP relies on the precise timing and order of inputs on either side of the synapse and thus constitutes a time-coding paradigm for plasticity induction ${ }^{19,20}$ by contrast to rate-coding plasticity protocols. The detection of a temporal coincidence between pre- and postsynaptic activities is crucial for STDP expression. Astrocytic glutamate uptake is involved in setting the timing of synaptic inputs. We therefore explored the role of EAAT2 in STDP, by transiently inhibiting (with DHK or WAY213,613) EAAT2 during STDP pairings. This allows an on-off manipulation compatible with STDP study, whereas genetic approaches (knockout) and long-lasting drug applications have potential long-term effects. DHK and WAY-213,613 have several advantages for studies of this type. In addition to their specificity for EAAT2 and their efficient washout, they are also nontransportable inhibitors of EAAT2, and this property prevents artificial increases in extracellular glutamate concentration due to hetero-exchange ${ }^{33,34}$. We next overexpressed EAAT2 with ceftriaxone, which has been reported to increase EAAT2 expression and activity 44 .

Astrocytic pools of EAAT2 are responsible for $90 \%$ of the glutamate uptake ${ }^{8}$. EAAT2 is also found on neurons but at much lower level ( $\sim 10 \%$ of astrocytic EAAT2). The physiological role of neuronal EAAT2 remains uncertain based on their very low level of expression but also on their distribution in most of the axon-terminal membranes and not being concentrated in the synapses ${ }^{9,56}$. Specific deletion of EAAT2 in astrocytes induces dramatic effects, such as excess mortality, lower body weight and spontaneous seizures, whereas no detectable neurological abnormalities are observed with neuronal EAAT2 deletion ${ }^{8,9}$.

The key feature of STDP is its occurrence within a restricted time window. Uncorrelated events $(>30 \mathrm{~ms})$ therefore fail to trigger plasticity. When EAAT2 activity is transiently impaired, an aberrant form of plasticity occurs during time windows in which plasticity is not normally observed. Uncorrelated events can induce this aberrant plasticity and are considered as pertinent events for an engram. Unlike STDP, the non-Hebbian LTP induced under transient EAAT2 blockade did not depend on the timing or order of pre- and postsynaptic activity. t-LTP 
has been reported to be mainly dependent on NMDARs ${ }^{19}$, which operate as molecular coincidence detectors ${ }^{4}$. By contrast, non-Hebbian LTP under EAAT2 blockade is dependent on postsynaptic GluN2B-containing NMDARs located extrasynaptically, and these receptors do not act as molecular coincident detectors. Supporting this, we found that even unpaired activity (consisting of a single postsynaptic action potential repeated 100 times at $1 \mathrm{~Hz}$ ) induced non-Hebbian LTP under EAAT2 blockade (Fig. 6g). Molecular coincidence detectors, such as NMDARs, require concomitant signals to be activated, as in STDP, in which the postsynaptic back-propagating action potential is paired with presynaptic activity ${ }^{19,20}$. In the presence of transient EAAT2 blockade, this feature is lost, because a single signal, the postsynaptic back-propagating action potential removing $\mathrm{Mg}^{2+}$ blockade, becomes sufficient to trigger LTP, due to the high ambient glutamate levels present when EAAT2 is blocked.

GABAergic microcircuits are involved in plasticity occurring at specific time window $\left(-70<\Delta t_{\mathrm{STDP}}<+70 \mathrm{~ms}\right)$ resulting in LTD (by contrast to the non-timing-dependent LTP). In the presence of DHK, GABAergic inhibition was stronger, due to the recruitment of inhibitory neurons as a result of the increase in glutamate spillover. In the presence of blockers of $\mathrm{GABA}_{\mathrm{A}} \mathrm{Rs}$ or VSCCs, pairings for which $-70<\Delta t_{\text {STDP }}<+70 \mathrm{~ms}$ unmasked NMDAR-mediated LTP.

We previously described the control of STDP polarity by $\mathrm{GABA}^{28}$. Here, different mechanisms are involved because concomitant transient blockade of GABAergic transmission and EAAT2 led to an absence of plasticity. GABAergic circuits are efficiently recruited by cortical stimulation in the presence of DHK. We hypothesize that the NMDAR-mediated LTP observed at large $\Delta t_{\text {STDP }}$ is somehow shunted at narrow $\Delta t_{\text {STDP }}$ by an additional pool of GABA, due to the recruitment of GABAergic interneurons by cortical stimulation. Indeed, NMDAR-mediated LTP at larger $\Delta t_{\text {STDP }}$ was exclusively dependent on the postsynaptic spiking (Fig. 6g) and did not require presynaptic stimulation. By contrast, when cortical stimulation (and, thus, the recruitment of GABAergic interneurons) was paired with the postsynaptic spike for narrow $\Delta t_{\mathrm{STDP}}$, the increased GABAergic transmission prevented LTP expression. Thus, NMDAR-mediated LTP may be expressed only at large $\Delta t_{\mathrm{STDP}}$, when presynaptic stimulation occurs far from the postsynaptic spike and GABAergic evoked transmission does not interfere with LTP expression. As a result, the blocking of $\mathrm{GABA}_{\mathrm{A}} \mathrm{R}$ transmission revealed LTP. This LTP was similar to the non-timing-dependent

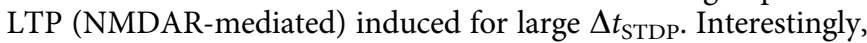
pre-post t-LTD and post-pre t-LTP observed in control conditions are both dependent on VSCC activity ${ }^{31}$, but their induction itself is not dependent on GABAergic transmission ${ }^{28}$. Thus, the t-LTD and t-LTP evoked in control conditions involve signalling mechanisms distinct from those involved in the plasticity observed under EAAT2 blockade.

EAAT2 overexpression by ceftriaxone prevented both t-LTP and t-LTD. We verified that ceftriaxone did not alter the passive and active electrophysiological properties of MSNs, as well as corticostriatal transmission and probability of glutamate release. Ceftriaxone can also mediate the upregulation of system $\mathrm{x}_{\mathrm{c}^{-}}(\text {cystine/glutamate antiporter system })^{57}$, which, together with EAAT2, is involved in the maintenance of glutamate homeostasis. However, the net effect of up or downregulation and the precise balance between these two systems (glutamate uptake and export) remains to be determined. We have previously shown that the bidirectional corticostriatal STDP relies on two distinct signalling pathways ${ }^{31,43}$. Indeed, t-LTP is NMDAR-dependent, whereas t-LTD is mGluR-mediated. Both receptor subtypes can be localized outside the synaptic cleft ${ }^{37,41}$ and thus compete with EAAT2 for the extracellular glutamate. Overexpression of EAAT2 by ceftriaxone is expected to enhance glutamate uptake and reduce spillover. This would reasonably result in a profound alteration of corticostriatal STDP expression. We hypothesize that enhanced glutamate clearance by EAAT2 upregulation may prevent the activation of postsynaptic NMDARs or typeImGluRs, leading to the lack of t-LTP or t-LTD, respectively. In line with that, increases in glutamate transporter expression have been shown to alter frequency-based plasticity dependent on glutamate spillover, such as mGluR-mediated LFS-LTD and HFSLTP in the hippocampus ${ }^{53}$. However, ceftriaxone has been mainly used in neurodegeneration and addiction models where extracellular glutamate levels are greatly enhanced ${ }^{10,12}$. Importantly, ceftriaxone abolishes the increase in glutamate spillover (assessed by NMDAR-EPSCs) in heroin-treated animals but not in control yoked saline animals ${ }^{58}$. In agreement with this ${ }^{58}$, we did not detect significant difference between NMDAR-EPSCs decay in saline and ceftriaxone-treated rats (Supplementary Fig. 5j-1). One possible explanation is that monitoring NMDAR-EPSCs does not allow differentiating between synaptic and extrasynaptic NMDARs. Therefore, the fraction of ambient versus synaptic glutamate detected by extrasynaptic NMDARs is difficult to assess. We hypothesize that under EAAT2 blockade, a critical number of peri- and/or extrasynaptic NMDARs are recruited leading to non-Hebbian plasticity. On the contrary, EAAT2 overexpression would reduce the pool of activated periand/or extrasynaptic NMDARs and consequently prevents STDP expression.

A few studies have reported effects of changes in EAAT2 expression on behaviour ${ }^{46}$. The pharmacological blockade of EAAT2 with DHK impairs spatial memory and induces depression and anhedonia and ceftriaxone has been reported to display antidepressant effects ${ }^{46}$. EAAT2 downregulation in striatum is also found in a rat model of depression ${ }^{59}$. EAAT2 $\mathrm{KO}$ mice exhibit seizures and premature death ${ }^{6,9}$. An inducible astrocytic EAAT2 knockout was recently shown to be associated with pathological repetitive behaviours and an increase in corticostriatal excitatory transmission ${ }^{25}$. Moreover, this phenotype was reversed by memantine treatment, confirming that excessive glutamate spillover due to EAAT2 dysfunction, deregulating the corticostriatal pathway, was responsible for the observed repetitive behaviours. These findings are consistent with our results showing that memantine prevents aberrant LTP in conditions of EAAT2 blockade. Conversely, EAAT2 overexpression has been reported to impair hippocampal learning ${ }^{60}$. This observation is consistent with our results showing a lack of plasticity with ceftriaxone treatment.

EAAT2 dysfunction, associated with higher ambient glutamate levels, has been observed in neurodegenerative and psychiatric diseases including Huntington's, Parkinson's, Alzheimer's and schizophrenia in which cognitive functions are impaired ${ }^{7,10,11}$ Chronic exposure to drugs of abuse has also been shown to induce a downregulation of EAAT2 in the nucleus accumbens ${ }^{12}$. EAAT2 therefore appears to be a major target for the treatment of neurological diseases and addiction (by ceftriaxone), not only to combat glutamatergic neurotoxicity but also to prevent aberrant plasticity, which could be linked to cognitive deficits ${ }^{10-12}$. Thus, our results, showing the tight control of STDP by EAAT2, are of importance for linking the expression of timing-dependent plasticity with different physiological or pathological states.

Astrocyte function is not restricted to structural and metabolic support or homeostatic and protective functions. Through glutamate uptake, astrocytes are also involved in higher brain functions, such as learning and memory $11,45,46$. We demonstrate here that EAAT2 operates over a highly controlled range to allow the emergence of bidirectional STDP. If STDP is dependent on the efficiency of glutamate uptake, then we would expect STDP 
expression to be controlled by the precise location and density of transporter expression, and glial synaptic coverage, which may differ considerably between brain structures and can undergo experience-dependent remodelling ${ }^{61}$ (Fig. 8). This work thus identifies astrocytes as key players in the establishment of synaptic Hebbian learning rule, such as STDP.

\section{Methods}

Animals. All experiments were performed in accordance with the guidelines of the local animal welfare committee (Center for Interdisciplinary Research in Biology Ethics Committee) and the EU (directive 2010/63/EU). Every precaution was taken to minimize stress and the number of animals used in each series of experiments. OFA rats P18-42 (Charles River, L'Arbresle, France) were used for brain slice electrophysiology. Animals were housed in standard 12-h light/dark cycles and food and water were available ad libitum.

Brain slice preparation. Horizontal brain slices containing the somatosensory cortical area and the corresponding corticostriatal projection field were prepared as previously described ${ }^{27,28,31}$. Corticostriatal connections (between somatosensory cortex layer 5 and the dorsolateral striatum) are preserved in the horizontal plane. Horizontal brain slices $(330 \mu \mathrm{m}$-thick) were prepared from rats with a vibrating blade microtome (VT1200S, Leica Micosystems, Nussloch, Germany). Brains were sliced in an ice-cold cutting solution $(125 \mathrm{mM} \mathrm{NaCl}, 2.5 \mathrm{mM} \mathrm{KCl}, 25 \mathrm{mM}$ glucose $25 \mathrm{mM} \mathrm{NaHCO}_{3}, 1.25 \mathrm{mM} \mathrm{NaH}_{2} \mathrm{PO}_{4}, 2 \mathrm{mM} \mathrm{CaCl}_{2}, 1 \mathrm{mM} \mathrm{MgCl}, 1 \mathrm{mM}$ pyruvic acid) through which $95 \% \mathrm{O}_{2} / 5 \% \mathrm{CO}_{2}$ was bubbled. The slices were transferred to the same solution at $34^{\circ} \mathrm{C}$ for $1 \mathrm{~h}$ and then to room temperature.

\section{Electrophysiology recordings. Patch-clamp recordings were performed as} previously described ${ }^{27,28,31}$. Briefly, for whole-cell recordings, borosilicate glass pipettes of 6-8 M $\Omega$ resistance were filled with (in $\mathrm{mM}$ ): $105 \mathrm{~K}$-gluconate, $30 \mathrm{KCl}$, 10 HEPES, 10 phosphocreatine, $4 \mathrm{Mg}$-ATP, $0.3 \mathrm{Na}$-GTP, 0.3 EGTA (adjusted to $\mathrm{pH} 7.35$ with $\mathrm{KOH})$. The composition of the extracellular solution was (mM): 125 $\mathrm{NaCl}, 2.5 \mathrm{KCl}, 25$ glucose, $25 \mathrm{NaHCO}_{3}, 1.25 \mathrm{NaH}_{2} \mathrm{PO}_{4}, 2 \mathrm{CaCl}_{2}, 1 \mathrm{MgCl}_{2}, 10 \mu \mathrm{M}$ pyruvic acid bubbled with $95 \% \mathrm{O}_{2}$ and $5 \% \mathrm{CO}_{2}$. Signals were amplified using with EPC9-2 and EPC10-4 amplifiers (HEKA Elektronik, Lambrecht, Germany). All recordings were performed at $34^{\circ} \mathrm{C}$, using a temperature control system (Bath-controller V, Luigs and Neumann, Ratingen, Germany) and slices were continuously superfused with extracellular solution, at a rate of $2 \mathrm{ml} \mathrm{min}^{-1}$. Slices were visualized under an Olympus BX51WI microscope (Olympus, Rungis, France), with a $4 \times / 0.13$ objective for the placement of the stimulating electrode and a $40 \times 10.80$ water-immersion objective for the localization of cells for wholecell recordings. Current-clamp recordings were filtered at $2.5 \mathrm{kHz}$ and sampled at $5 \mathrm{kHz}$ and voltage-clamp recordings were filtered at $5 \mathrm{kHz}$ and sampled at $10 \mathrm{kHz}$, with the Patchmaster v2 $\times 32$ program (HEKA Elektronik).

To compare the decay time of NMDAR-mediated EPSCs in saline- and ceftriaxone-treated animals, whole-cell recordings in voltage-clamp mode were performed at $+40 \mathrm{mV}$ clamping voltage. Borosilicate glass pipettes were filled with (in $\mathrm{mM}$ ): 124 cesium, $10 \mathrm{NaCl}, 1 \mathrm{MgCl}_{2}, 10$ HEPES, 1 EGTA, 1 QX, $2 \mathrm{Mg}$-ATP, $0.3 \mathrm{Na}$-GTP (adjusted to $\mathrm{pH} 7.35$ with $\mathrm{CsOH}$ ). The stimulation protocol used for triggering NMDAR-EPSCs consisted in eight presynaptic stimulation pulses elicited at $100 \mathrm{~Hz}$.

\section{Spike timing-dependent plasticity protocols and random $\Delta t_{\text {STDP }}$ patterns.} Electrical stimulations were performed with a concentric bipolar electrode (Phymep, Paris, France and CBBSE75 FHC, Bowdoin, ME, USA) placed in layer 5 of the somatosensory cortex ${ }^{27}$. Electrical stimulations were monophasic, at constant current (ISO-Flex stimulator, AMPI, Jerusalem, Israel). Currents were adjusted to evoke 100-400 pA EPSCs. Repetitive control stimuli were applied at $0.1 \mathrm{~Hz}$. STDP protocols consisted of pairings of pre- and postsynaptic stimulations (at $1 \mathrm{~Hz})$ separated by a specific time interval $\left(\Delta t_{\mathrm{STDP}}\right)$. Presynaptic stimulations corresponded to cortical stimulations and the postsynaptic stimulation of an action potential evoked by a depolarizing current step (30 ms duration) in MSNs. $\Delta t_{\mathrm{STDP}}<0 \mathrm{~ms}$ for post-pre pairings, and $\Delta t_{\mathrm{STDP}}>0 \mathrm{~ms}$ for pre-post pairings. $\Delta t_{\mathrm{STDP}}= \pm 500 \mathrm{~ms}$ corresponds to post-pre and pre-post pairings performed around $\Delta t_{\mathrm{STDP}}=-500 \mathrm{~ms}$ and $\Delta t_{\mathrm{STDP}}=+500 \mathrm{~ms}$. Note that for

$\Delta t_{\mathrm{STDP}}=-500 \mathrm{~ms}$ and $\Delta t_{\mathrm{STDP}}=+500 \mathrm{~ms}$, the order (post-pre versus pre-post) was determined by the first pairing of the STDP protocol only, because, for the remaining pairings, the pre- and postsynaptic stimulations were separated by $500 \mathrm{~ms}$ and could therefore be considered as either post-pre or pre-post pairings at $1 \mathrm{~Hz}$. We therefore pooled the data for $\Delta t_{\mathrm{STDP}}=-500 \mathrm{~ms}$ and $\Delta t_{\mathrm{STDP}}=+500 \mathrm{~ms}$ $\left(\Delta t_{\mathrm{STDP}}= \pm 500 \mathrm{~ms}\right)$, which are presented as a single average on the figures. Recordings on neurons were made over a period of $10 \mathrm{~min}$ at baseline, and for at least $50 \mathrm{~min}$ after the SDTP protocols; long-term changes in synaptic efficacy were measured for the last $10 \mathrm{~min}$. We individually measured and averaged 60 successive EPSCs, comparing the last $10 \mathrm{~min}$ of the recording with the 10 -minute baseline recording. Whole-cell recordings were made in voltage-clamp mode during baseline and for the $60 \mathrm{~min}$ of recording after the STDP protocol, and in current- clamp mode during STDP protocol. Experiments were excluded if input resistance (Ri) varied by more than $20 \%$.

For the random $\Delta t_{\text {STDP }}$ patterns, we used the following algorithm (programmed in Igor Pro 6.3 software, WaveMetrics): for each pairing, we first selected a time window with a randomly selected length between 500 and 1,500 ms (with a uniform distribution) and located the presynaptic stimulation time in the middle of this window. The postsynaptic stimulation time was then randomly chosen within this window (with a uniform distribution). The $\Delta t_{\text {STDP }}$ pattern was formed by the concatenation of 100 such windows. This generated both a close-touniform distribution of the $\Delta t_{\mathrm{STDP}}$ and a variable interval between two successive presynaptic stimulations.

Chemicals. All chemicals were purchased from Tocris (Ellisville, MO, USA), except for picrotoxin (Sigma). (2S,3S,4R)-2-Carboxy-4-isopropyl-3-pyrrolidineacetic acid (Dihydrokainic acid, DHK; $300 \mu \mathrm{M}$ ), DL-2-amino-5-phosphono-pentanoic acid (D-AP5; $50 \mu \mathrm{M}),(1 \mathrm{~S}, 2 \mathrm{~S})-2-[2-[[3-(1 \mathrm{H}-$ benzimidazol2yl)propyl]methylamino] ethyl]-6-fluoro-1,2,3,4-tetrahydro-1-(1-methylethyl)-2naphthalenyl methoxyacetoacetate dihydrochloride (Mibefradil; $20 \mu \mathrm{M}$ ), 6-cyano7-nitroquinoxaline-2,3-dione (CNQX; $20 \mu \mathrm{M}),(\alpha \mathrm{R}, \beta \mathrm{S})$ - $\alpha$-(4-hydroxyphenyl)- $\beta$ methyl-4-(phenylmethyl)-1-piperidinepropanol maleate (Ro 25-6981;10 $\mu \mathrm{M}$ ), SR 95531 hydrobromide (gabazine; $10 \mu \mathrm{M}$ ) and 3,5-dimethyl-tricyclo[3.3.1.13,7]decan-1-amine hydrochloride (Memantine; $10 \mu \mathrm{M}$ ) were dissolved directly in the extracellular solution and bath applied. N-(piperidin-1-yl)-5-(4-iodophenyl)-1(2,4-dichlorophenyl)-4-methyl-1H-pyrazole-3-carboxamide (AM251;3 $\mu \mathrm{M}$ ) and picrotoxin $(50 \mu \mathrm{M})$ were dissolved in ethanol and added to the external solution, such that the final concentration of ethanol was $0.01-0.1 \%$. N-[4-(2-bromo-4,5difluorophenoxy)phenyl]-L-asparagine (WAY-213,613; 50 and $100 \mu \mathrm{M}$ ) was dissolved in DMSO and added to the external solution such that the final concentration of DMSO was 0.05 and $0.1 \%$, respectively. (S)- $\alpha$-Methyl-4-carboxyphenylglycine (MCPG; $500 \mu \mathrm{M})$ was dissolved in 1.1 eq. $\mathrm{NaOH}$ and added to the external solution. BAPTA $(10 \mathrm{mM})$ and dizocilpine maleate (i-MK801; $1 \mathrm{mM})$ were dissolved directly in the intracellular solution.

The contrasting activity patterns of synaptic and extrasynaptic NMDARs result in different degrees of memantine blockade ${ }^{42}$. Due to the agonist concentrationdependence of memantine blockade kinetics, slices were pre-incubated with low dose of memantine $(10 \mu \mathrm{M})$ for at least $1 \mathrm{~h}$ before recording, to allow sufficient time for equilibrium to be reached.

Transient EAAT2 blockade. Transient EAAT2 blockade was achieved with two structurally different molecules: DHK $(300 \mu \mathrm{M})^{33}$ and WAY-213,613 $(50-100 \mu \mathrm{M})^{34}$, which are both selective non-substrate inhibitors

(non-transportable) of EAAT2. DHK was bath-applied for as short a period as possible, to ensure that its effect on $\mathrm{Vm}$ was compatible with the correct analysis of synaptic efficacy changes. Indeed, EAAT2 blockade resulted in a marked depolarization $^{26}$, potentially impairing the estimation of synaptic efficacy changes. A stable baseline was established over a period of $10 \mathrm{~min}$. DHK was bath-applied for $5 \mathrm{~min}$ (the dark gray area in the figures). We systematically checked the efficacy of DHK application before applying the STDP protocol. This depolarization (Fig. 2a) was used as an indicator of DHK efficiency. DHK was washed out at the STDP protocol offset. The full DHK washout took $15 \mathrm{~min}$ (the light gray area in the figures) and, during this period, a significant and transient decrease in EPSC magnitude (due to the DHK-induced inward shift in $I_{\text {holding }}$ and AMPAR desensitization ${ }^{26}$ ) was observed. Accordingly, in all figures, synaptic efficacy changes are illustrated from 15 min after the removal of DHK. Synaptic efficacy changes were evaluated 50-60 min after the start of the DHK washout (at least $30 \mathrm{~min}$ after the full recovery of baseline $I_{\text {holding }}$ ).

Electrophysiological data analysis. Off-line analysis was performed with Fitmaster (Heka Elektronik). Spontaneous post-synaptic currents (sPSCs) were identified using a semi-automated amplitude threshold based detection software (Mini Analysis 6.0.7 Program, Synaptosoft, Fort Lee, NJ, USA) and were visually confirmed. Analysis of NMDAR-EPSCs was performed using a custom-build analysis in Python. After removal of the stimulation artifacts, NMDAR-EPSCs decay was normalized and fitted to a bi-exponential curve. The fast and slow decay times of NMDAR-EPSCs (tau1 and tau2, respectively) were then quantified. Statistical analysis was performed with Prism 5.02 software (San Diego, CA, USA). In all cases ' $n$ ' refers to an experiment on a single cell from a single slice. In average, 2 cells per animal were obtained. All results are expressed as mean \pm s.e.m. in the text and in the figures (except as mean \pm s.d. in the figures for plasticity graphs: normalized EPSC versus time), and statistical significance was assessed in unpaired $t$-tests or in one-sample $t$-tests, as appropriate, using the indicated significance threshold $(P)$, or one-way ANOVA with Bonferroni correction, where specified.

Chronic ceftriaxone treatment. To increase the expression of EAAT2 chronic ceftriaxone treatment of the rats was performed as previously described ${ }^{53}$. Male OFA rats (P30-P42) received a daily intraperitoneal (i.p.) injection of ceftriaxone (Rocefin, Roche; $200 \mathrm{mg} \mathrm{kg}^{-1}$ per day dissolved in saline) or an equal volume of saline on eight consecutive days. Corticostriatal brain slices for electrophysiology 
were obtained from ceftriaxone- or saline-treated rats $24 \mathrm{~h}$ after the final injection, and prepared as described above.

Immunohistochemistry. Rats were treated for eight days with daily i.p. injection of either saline ( $n=4$ rats) or ceftriaxone ( $n=4$ rats), as described above. Rats were anesthetized with pentobarbital. Brains were postfixed in $4 \%$ paraformaldehyde and cut into $30 \mu \mathrm{m}$ horizontal sections with a vibratome (Microm HM650V, ThermoScientific). Immunostaining was performed by incubating free-floating sections with a guinea pig anti-EAAT2 antibody (1:5000; AB1783, Merck Millipore) for $48 \mathrm{~h}$ at $4{ }^{\circ} \mathrm{C}$ and then with a secondary Cyanine Cy3-conjugated antibody (1:1,000; Jackson Laboratories) dissolved in PBS $1 \mathrm{X}$ for $1 \mathrm{~h}$. Images were acquired with the SP5 confocal system (Leica, Germany).

Data availability. All relevant data are available from the authors.

\section{References}

1. Diamond, J. S. \& Jahr, C. E. Transporters buffer synaptically released glutamate on a submillisecond time scale. J. Neurosci. 17, 4672-4687 (1997).

2. Kullmann, D. M. \& Asztely, F. Extrasynaptic glutamate spillover in the hippocampus: evidence and implications. Trends Neurosci. 21, 8-14 (1998).

3. Takahashi, M. et al. The role of glutamate transporters in glutamate homeostasis in the brain. J. Exp. Biol. 200(Pt 2): 401-409 (1997).

4. Attwell, D. \& Gibb, A. Neuroenergetics and the kinetic design of excitatory synapses. Nat. Rev. Neurosci. 6, 841-849 (2005).

5. Rothstein, J. D. et al. Knockout of glutamate transporters reveals a major role for astroglial transport in excitotoxicity and clearance of glutamate. Neuron 16, 675-686 (1996).

6. Tanaka, K. et al. Epilepsy and exacerbation of brain injury in mice lacking the glutamate transporter GLT-1. Science 276, 1699-1702 (1997).

7. Danbolt, N. C. Glutamate uptake. Prog. Neurobiol. 65, 1-105 (2001).

8. Danbolt, N. C., Furness, D. N. \& Zhou, Y. Neuronal vs glial glutamate uptake: resolving the conundrum. Neurochem. Int. 98, 29-45 (2016).

9. Petr, G. T. et al. Conditional deletion of the glutamate transporter GLT-1 reveals that astrocytic GLT-1 protects against fatal epilepsy while neuronal GLT-1 contributes significantly to glutamate uptake into synaptosomes. J. Neurosci.. 1 35, 5187-5201 (2015).

10. Soni, N., Reddy, B. V. \& Kumar, P. GLT-1 transporter: An effective pharmacological target for various neurological disorders. Pharmacol. Biochem. Behav. 127C, 70-81 (2014).

11. Chung, W. S., Welsh, C. A., Barres, B. A. \& Stevens, B. Do glia drive synaptic and cognitive impairment in disease? Nat. Neurosci. 18, 1539-1545 (2015).

12. Scofield, M. D. \& Kalivas, P. W. Astrocytic dysfunction and addiction: consequences of impaired glutamate homeostasis. Neuroscientist 20, 610-622 (2014).

13. Tzingounis, A. V. \& Wadiche, J. I. Glutamate transporters: confining runaway excitation by shaping synaptic transmission. Nat. Rev. Neurosci. 8, 935-947 (2007).

14. Arnth-Jensen, N., Jabaudon, D. \& Scanziani, M. Cooperation between independent hippocampal synapses is controlled by glutamate uptake. Nat. Neurosci. 5, 325-331 (2002).

15. Scimemi, A., Fine, A., Kullmann, D. M. \& Rusakov, D. A. NR2B-containing receptors mediate cross talk among hippocampal synapses. J. Neurosci. 24, 4767-4777 (2004).

16. Diamond, J. S. \& Jahr, C. E. Synaptically released glutamate does not overwhelm transporters on hippocampal astrocytes during high-frequency stimulation. J. Neurophysiol. 83, 2835-2843 (2000).

17. Asztely, F., Erdemli, G. \& Kullmann, D. M. Extrasynaptic glutamate spillover in the hippocampus: dependence on temperature and the role of active glutamate uptake. Neuron 18, 281-293 (1997).

18. Bergles, D. E. \& Jahr, C. E. Synaptic activation of glutamate transporters in hippocampal astrocytes. Neuron 19, 1297-1308 (1997).

19. Feldman, D. E. The spike-timing dependence of plasticity. Neuron 75, 556-571 (2012).

20. Sjöström, P. J., Rancz, E. A., Roth, A. \& Häusser, M. Dendritic excitability and synaptic plasticity. Physiol. Rev. 88, 769-840 (2008).

21. Clopath, C., Büsing, L., Vasilaki, E. \& Gerstner, W. Connectivity reflects coding: a model of voltage-based STDP with homeostasis. Nat. Neurosci. 13, 344-352 (2010).

22. Costa, R. P., Froemke, R. C., Sjöström, P. J. \& van Rossum, M. C. Unified preand postsynaptic long-term plasticity enables reliable and flexible learning. Elife 26, 4 (2015).

23. Di Filippo, M. et al. Short-term and long-term plasticity at corticostriatal synapses: implications for learning and memory. Behav. Brain Res. 199, 108-118 (2009).

24. Yin, H. H. et al. Dynamic reorganization of striatal circuits during the acquisition and consolidation of a skill. Nat. Neurosci. 12, 333-341 (2009).
25. Aida, T. et al. Astroglial glutamate transporter deficiency increases synaptic excitability and leads to pathological repetitive behaviors in mice. Neuropsychopharmacology 40, 1569-1579 (2015).

26. Goubard, V., Fino, E. \& Venance, L. Contribution of astrocytic glutamate and GABA uptake to corticostriatal information processing. J. Physiol. 589(Pt 9): 2301-2319 (2011).

27. Fino, E., Glowinski, J. \& Venance, L. Bidirectional activity-dependent plasticity at corticostriatal synapses. J. Neurosci. 25, 11279-11287 (2005).

28. Paillé, V. et al. GABAergic circuits control spike-timing-dependent plasticity. J. Neurosci.. 29 33, 9353-9363 (2013).

29. Pawlak, V. \& Kerr, J. N. Dopamine receptor activation is required for corticostriatal spike-timing-dependent plasticity. J. Neurosci. 28, 2435-2446 (2008).

30. Shen, W., Flajolet, M., Greengard, P. \& Surmeier, D. J. Dichotomous dopaminergic control of striatal synaptic plasticity. Science 321, 848-851 (2008).

31. Fino, E. et al. Distinct coincidence detectors govern the corticostriatal spike timing-dependent plasticity. J. Physiol. 588, 3045-3062 (2010).

32. Schulz, J. M., Redgrave, P. \& Reynolds, J. N. Cortico-striatal spike-timing dependent plasticity after activation of subcortical pathways. Front Synaptic Neurosci 2, 23 (2010).

33. Arriza, J. L. et al. Functional comparisons of three glutamate transporter subtypes cloned from human motor cortex. J. Neurosci. 14, 5559-5569 (1994).

34. Dunlop, J. et al. Characterization of novel aryl-ether, biaryl, and fluorene aspartic acid and diaminopropionic acid analogs as potent inhibitors of the high-affinity glutamate transporter EAAT2. Mol. Pharmacol. 68, 974-982 (2005).

35. Artola, A., Bröcher, S. \& Singer, W. Different voltage-dependent thresholds for inducing long-term depression and long-term potentiation in slices of rat visual cortex. Nature 347, 69-72 (1990).

36. Lei, S. et al. Depolarization-induced long-term depression at hippocampal mossy fiber-CA3 pyramidal neuron synapses. J. Neurosci. 23, 9786-9795 (2003).

37. Baude, A. et al. The metabotropic glutamate receptor (mGluR1 alpha) is concentrated at perisynaptic membrane of neuronal subpopulations as detected by immunogold reaction. Neuron 11, 771-787 (1993).

38. Huang, Y. H., Sinha, S. R., Tanaka, K., Rothstein, J. D. \& Bergles, D. E. Astrocyte glutamate transporters regulate metabotropic glutamate receptormediated excitation of hippocampal interneurons. J. Neurosci. 24, 4551-4559 (2004).

39. Lozovaya, N. A. et al. Extrasynaptic NR2B and NR2D subunits of NMDA receptors shape 'superslow' afterburst EPSC in rat hippocampus. J. Physiol. 15558(Pt 2): 451-463 (2004).

40. Chalifoux, J. R. \& Carter, A. G. Glutamate spillover promotes the generation of NMDA spikes. J. Neurosci. 31, 16435-16446 (2011).

41. Paoletti, P., Bellone, C. \& Zhou, Q. NMDA receptor subunit diversity: impact on receptor properties, synaptic plasticity and disease. Nat. Rev. Neurosci. 14, 383-400 (2013).

42. Xia, P., Chen, H. S., Zhang, D. \& Lipton, S. A. Memantine preferentially blocks extrasynaptic over synaptic NMDA receptor currents in hippocampal autapses. J. Neurosci. 30, 11246-11250 (2010).

43. Evans, R. C. et al. The effects of NMDA subunit composition on calcium influx and spike timing-dependent plasticity in striatal medium spiny neurons. PLoS Comput. Biol. 8, e1002493 (2012).

44. Rothstein, J. D. et al. Beta-lactam antibiotics offer neuroprotection by increasing glutamate transporter expression. Nature 433, 73-77 (2005).

45. Fields, R. D. et al. Glial biology in learning and cognition. Neuroscientist 20, 426-431 (2014).

46. Oliveira, J. F., Sardinha, V. M., Guerra-Gomes, S., Araque, A. \& Sousa, N. Do stars govern our actions? Astrocyte involvement in rodent behavior. Trends Neurosci. 38, 535-549 (2015).

47. Min, R. \& Nevian, T. Astrocyte signaling controls spike timing-dependent depression at neocortical synapses. Nat. Neurosci. 15, 746-753 (2012).

48. Katagiri, H., Tanaka, K. \& Manabe, T. Requirement of appropriate glutamate concentrations in the synaptic cleft for hippocampal LTP induction. Eur J. Neurosci. 14, 547-553 (2001).

49. Pinard, A., Lévesque, S., Vallée, J. \& Robitaille, R. Glutamatergic modulation of synaptic plasticity at a PNS vertebrate cholinergic synapse. Eur J. Neurosci. 18, 3241-3250 (2003).

50. Massey, P. V. et al. Differential roles of NR2A and NR2B-containing NMDA receptors in cortical long-term potentiation and long-term depression. J. Neurosci. 24, 7821-7828 (2004).

51. Tsvetkov, E., Shin, R. M. \& Bolshakov, V. Y. Glutamate uptake determines pathway specificity of long-term potentiation in the neural circuitry of fear conditioning. Neuron 41, 139-151 (2004). 
52. Wang, Z. Y., Zhang, Y. Q. \& Zhao, Z. Q. Inhibition of tetanically sciatic stimulation-induced LTP of spinal neurons and Fos expression by disrupting glutamate transporter GLT-1. Neuropharmacology 51, 764-772 (2006).

53. Omrani, A. et al. Up-regulation of GLT-1 severely impairs LTD at mossy fiberCA3 synapses. J. Physiol. 587(Pt 19): 4575-4588 (2009).

54. Scimemi, A., Tian, H. \& Diamond, J. S. Neuronal transporters regulate glutamate clearance, NMDA receptor activation, and synaptic plasticity in the hippocampus. J. Neurosci. 29, 14581-14595 (2009).

55. Wadiche, J. I. \& Jahr, C. E. Patterned expression of Purkinje cell glutamate transporters controls synaptic plasticity. Nat. Neurosci. 8, 1329-1334 (2005).

56. Furness, D. N. et al. A quantitative assessment of glutamate uptake into hippocampal synaptic terminals and astrocytes: new insights into a neuronal role for excitatory amino acid transporter 2 (EAAT2). Neuroscience 11157, 80-94 (2008).

57. Lewerenz, J. et al. Induction of Nrf2 and xCT are involved in the action of the neuroprotective antibiotic ceftriaxone in vitro. J. Neurochem. 111, 332-343 (2009).

58. Shen, H., Scofield, M. D., Boger, H., Hensley, M. \& Kalivas, P. W. Synaptic glutamate spillover due to impaired glutamate uptake mediates heroin relapse. J. Neurosci. 34, 5649-5657 (2014).

59. Almeida, R. F. et al. Effects of depressive-like behavior of rats on brain glutamate uptake. Neurochem. Res. 35, 1164-1171 (2010).

60. Matos-Ocasio, F., Hernández-López, A. \& Thompson, K. J. Ceftriaxone, a GLT1 transporter activator, disrupts hippocampal learning in rats. Pharmacol. Biochem. Behav. 122, 118-121 (2014).

61. Bernardinelli, Y., Muller, D. \& Nikonenko, I. Astrocyte-synapse structural plasticity. Neural. Plast. 2014, 232105 (2014).

\section{Acknowledgements}

We thank the members of the LV laboratory, Hugues Berry, Ilya Prokin, Olivier Manzoni and Christian Giaume, for helpful suggestions and critical comments. We thank Giuseppe Gangarossa and Sylvie Perez for assistance with immunohistochemistry.
S.V. is a Research Fellow of the MRT and the LabEx MemoLife. This work was supported by grants from INSERM, Collège de France, CNRS and Fondation de France.

\section{Author contributions}

S.V. and L.V. conceived and designed the experiments; S.V. performed experiments and analysis; S.V. and L.V. interpreted data and wrote the manuscript; L.V. supervised the project.

\section{Additional information}

Supplementary Information accompanies this paper at http://www.nature.com/ naturecommunications

Competing financial interests: The authors declare no competing financial interests.

Reprints and permission information is available online at http://npg.nature.com/ reprintsandpermissions/

How to cite this article: Valtcheva, S. \& Venance, L. Astrocytes gate Hebbian synaptic plasticity in the striatum. Nat. Commun. 7, 13845 doi: 10.1038/ncomms13845 (2016).

Publisher's note: Springer Nature remains neutral with regard to jurisdictional claims in published maps and institutional affiliations.

\section{(c) ()}

This work is licensed under a Creative Commons Attribution 4.0 International License. The images or other third party material in this article are included in the article's Creative Commons license, unless indicated otherwise in the credit line; if the material is not included under the Creative Commons license, users will need to obtain permission from the license holder to reproduce the material To view a copy of this license, visit http://creativecommons.org/licenses/by/4.0/

(C) The Author(s) 2016 